TRANSACTIONS OF THE

AMERICAN MATHEMATICAL SOCIETY

Volume 355, Number 8, Pages 3301-3328

S 0002-9947(03)03142-8

Article electronically published on April 25, 2003

\title{
STABILITY OF INFINITE-DIMENSIONAL SAMPLED-DATA SYSTEMS
}

\author{
HARTMUT LOGEMANN, RICHARD REBARBER, AND STUART TOWNLEY
}

\begin{abstract}
Suppose that a static-state feedback stabilizes a continuous-time linear infinite-dimensional control system. We consider the following question: if we construct a sampled-data controller by applying an idealized sample-andhold process to a continuous-time stabilizing feedback, will this sampled-data controller stabilize the system for all sufficiently small sampling times? Here the state space $X$ and the control space $U$ are Hilbert spaces, the system is of the form $\dot{x}(t)=A x(t)+B u(t)$, where $A$ is the generator of a strongly continuous semigroup on $X$, and the continuous time feedback is $u(t)=F x(t)$. The answer to the above question is known to be "yes" if $X$ and $U$ are finitedimensional spaces. In the infinite-dimensional case, if $F$ is not compact, then it is easy to find counterexamples. Therefore, we restrict attention to compact feedback. We show that the answer to the above question is "yes", if $B$ is a bounded operator from $U$ into $X$. Moreover, if $B$ is unbounded, we show that the answer "yes" remains correct, provided that the semigroup generated by $A$ is analytic. We use the theory developed for static-state feedback to obtain analogous results for dynamic-output feedback control.
\end{abstract}

\section{INTRODUCTION}

Consider the following system with state space $X$ and input space $U$ (both Hilbert spaces):

$$
\dot{x}(t)=A x(t)+B u(t), \quad x(0)=x^{0} \in X,
$$

where $A$ is the generator of a strongly continuous semigroup $T(t)$ on $X$ and $B$ is a bounded linear operator from $U$ into $X_{-1}$, where $X_{-1}$ is a certain extrapolation space with $X \hookrightarrow X_{-1}$. If the input (or control) operator $B$ maps boundedly into the state space $X$, then $B$ is called bounded; otherwise $B$ is called "unbounded" (with respect to the state space $X$ ). We give precise details of the set-up in Section 2 .

We consider the following fundamental problem: Suppose that the feedback control $u(t)=F x(t)$, where $F$ is a bounded linear operator from $X$ into $U$, is an exponentially stabilizing state feedback control for (1.1) in the sense that $A+B F$ generates an exponentially stable strongly continuous semigroup on $X$. A natural implementation of this continuous-time control $u(t)=F x(t)$ is to use sample and

Received by the editors December 21, 2000 and, in revised form, February 21, 2002.

2000 Mathematics Subject Classification. Primary 34G10, 47A55, 47D06, 93C25, 93C57, $93 \mathrm{D} 15$.

This work was supported by NATO (Grant CRG 950179) and by the NATIONAL SCIENCE Foundation (Grant DMS-9623392). 
hold, so that $u$ is given instead by

$$
u(t)=F x(k \tau), \quad t \in[k \tau,(k+1) \tau) .
$$

Here $\tau>0$ is the sampling period and $t=n \tau, n=0,1, \ldots$, are the sampling times. The control (1.2) is called a sampled-data feedback control and the overall system given by (1.1) and (1.2) is called a sampled-data feedback system. Intuitively, we would expect that for all sufficiently small sampling periods $\tau>0$, (1.2) produces a stabilizing control for (1.1) in the sense that there exist $N \geq 1$ and $\nu>0$ so that

$$
\|x(t)\| \leq N e^{-\nu t}\left\|x^{0}\right\|, \quad \forall x^{0} \in X, \forall t \geq 0 .
$$

Integrating (1.1) and (1.2) over one sampling interval $[k \tau,(k+1) \tau)$ and setting $x_{k}:=x(k \tau)$ yields the following discrete-time system:

$$
x_{k+1}=\Delta_{\tau} x_{k}, \quad \text { where } \Delta_{\tau}:=T(\tau)+\int_{0}^{\tau} T(s) B F d s .
$$

It is straightforward to show that $\Delta_{\tau}$ is a bounded operator from $X$ to $X$ and that (1.3) holds if, and only if, $\Delta_{\tau}$ is power stable, i.e., $\left\|\Delta_{\tau}^{k}\right\| \rightarrow 0$ as $k \rightarrow \infty$ (see Section 2 ). Then the fundamental problem described above becomes that of determining whether or not $\Delta_{\tau}$ is power stable for all sufficiently small $\tau>0$.

By way of motivation, let us briefly discuss the finite-dimensional case. Then $X=\mathbb{R}^{n}, U=\mathbb{R}^{m}, A, B$ and $F$ are real matrices of compatible formats and $T(t)=e^{A t}$. It is instructive to sketch a simple argument showing in this case that the sampled-data feedback (1.2) is indeed stabilizing for (1.1), provided that $\tau$ is sufficiently small. Since $u(t)=F x(t)$ is an exponentially stabilizing static state feedback control for (1.1), the eigenvalues of $A+B F$ have negative real parts, or, equivalently, there exists a positive definite symmetric matrix $P$ satisfying

$$
(A+B F)^{T} P+P(A+B F)=-I .
$$

Next we observe, using the power series expansion of the matrix exponential $e^{A t}$, that there exists $M_{1} \geq 0$ so that

$$
\left\|\Delta_{\tau}-I-\tau(A+B F)\right\| \leq M_{1} \tau^{2}, \quad \forall \tau \in(0,1] .
$$

Then, invoking (1.4), it is easy to see that there exist $M_{2}>0$ and $\tau^{*}>0$ such that $x^{T} \Delta_{\tau}^{T} P \Delta_{\tau} x-x^{T} P x \leq 2 \tau x^{T}(A+B F) P x+M_{2} \tau^{2}\|x\|^{2} \leq-\frac{1}{2} \tau\|x\|^{2}, \forall \tau \in\left(0, \tau^{*}\right)$.

Therefore, for every $\tau \in\left(0, \tau^{*}\right), \Delta_{\tau}^{T} P \Delta_{\tau}-P<0$, or, equivalently, $\Delta_{\tau}$ is power stable, showing that the sampled-data feedback (1.2) is stabilizing for all sufficiently small $\tau>0$. A different proof of this result can be found in Chen and Francis [1, where, using the power series expansion of the matrix exponential and the continuous dependence of the eigenvalues of $\Delta_{\tau}$ on $\tau$, it is shown that the spectral radius of $\Delta_{\tau}$ is less than one for all sufficiently small $\tau>0$ (which implies powerstability of $\Delta_{\tau}$ for all suffciently small $\tau>0$ ).

Whilst this simple observation is easy to prove and well known in the finitedimensional control literature, it serves to indicate that extending the above result to infinite-dimensional situations is far from trivial. Indeed, neither the proof sketched above nor the proof given in [1] have meaningful generalisations to the infinite-dimensional case, because they both rely on the convergence of the Taylor series of $e^{A t}$ in the operator-norm topology, which extends to the infinitedimensional case only if the generator of the semigroup is bounded, or, equivalently, 
if the semigroup is uniformly continuous. Systems described by uniformly continuous semigroups are not general enough to encompass most interesting examples.

It might appear that the case considered above of sampling a static state or output feedback control, whilst mathematically significant and simple to describe, is too restrictive for meaningful applications. However, results for the more practically relevant case of sampling a dynamic output feedback control can be obtained from the state feedback case quite easily, as we show in Section 5 (in an infinitedimensional context). Such sampling of dynamic continuous-time controllers forms the basis for a so-called indirect sampled-data design methodology (see Chen and Francis [2] and Franklin, Powell and Workman [6] for a fuller description of this methodology in the finite-dimensional case).

Recently there has been increasing interest in sampled-data control of infinitedimensional systems; see Rebarber and Townley [15], [16], Rosen and Wang [18] and Tarn et al. 20. From these papers a trend emerges: if the underlying semigroup system is analytic, or the input operator is bounded, then typically the finitedimensional sampled-data result generalises to the infinite-dimensional case; if the input operator is unbounded and the underlying semigroup is not analytic, then the generalisation fails. It is not surprising that analyticity plays such a key role. In some sense, analyticity constrains the spectrum of the generator of the underlying semigroup so as to avoid any losses of high-frequency information when sampling.

We give a brief description of the results contained in this paper. In Section 2 we introduce the class of infinite-dimensional systems to which our results apply and we present some preliminary basic technical results to be used in the rest of the paper. In Section 3 we consider the case in which the input operator $B$ is bounded, but the semigroup generated by $A$ is not assumed to be analytic. We show that if the feedback operator $F$ is compact and the semigroup generated by $A+B F$ is exponentially stable, then, for sufficiently small $\tau>0$, the sampled-data feedback (1.2) is stabilizing. A key element of the proof is the introduction of a new, equivalent norm on the state space with respect to which the operator $\Delta_{\tau}$ is a strict contraction, provided that $\tau$ is sufficiently small. We also give a simple example which shows that the result is not true if the compactness assumption on $F$ is removed. In Section 4 we consider the case in which the input operator $B$ is unbounded and the semigroup generated by $A$ is analytic. We show that for any compact feedback operator $F$, the operator $A+B F$ (with suitable domain) generates an analytic semigroup on the state space $X$, and if this semigroup is exponentially stable, then the sampled-data feedback (1.2) is stabilizing for all sufficiently small $\tau>0$. The proof of this result is quite involved and differs considerably from the argument leading to the result for bounded $B$. Instead of trying to estimate the spectral radius of $\Delta_{\tau}$ directly, we invoke frequency-domain stability criteria for the power stability of $\Delta_{\tau}$, making repeated use of the analyticity of the underlying semigroup. Finally, in Section 5 we show how to use the static state feedback results of Sections 3 and 4 in the practically more relevant situation of sampling a dynamic output feedback controller.

Notation: For $\alpha \in \mathbb{R}, r>0$ and $z \in \mathbb{C}$, we define

$$
\mathbb{C}_{\alpha}:=\{s \in \mathbb{C} \mid \operatorname{Re} s>\alpha\}, \quad \mathbb{B}(z, r):=\{s \in \mathbb{C}|| s-z \mid<r\} .
$$

The exterior of the unit disc is denoted by $\mathbb{E}$, i.e.,

$$
\mathbb{E}:=\{s \in \mathbb{C}|| s \mid>1\} .
$$


Let $X$ and $Y$ be Banach spaces. The space of all bounded linear operators from $X$ to $Y$ is denoted by $\mathcal{B}(X, Y)$; we set $\mathcal{B}(X):=\mathcal{B}(X, X)$. Let $A$ be an operator with domain and range in $X$. Then $D(A)$ denotes the domain of $A$ and $\varrho(A)$ denotes the resolvent set of $A$, i.e., the set of all $s \in \mathbb{C}$ such that $s I-A: D(A) \rightarrow X$ is bijective and $(s I-A)^{-1} \in \mathcal{B}(X)$. The spectrum of $A$, which is the complement of $\varrho(A)$, is denoted by $\sigma(A)$. Let $\sigma_{p}(A) \subset \sigma(A)$ denote the point spectrum (i.e., the set of all eigenvalues) of $A$. If $S \subset X$ and $A: D(A) \subset X \rightarrow Y$ is an operator, then $\left.A\right|_{S}$ denotes the restriction of $A$ to $S$, i.e., the operator defined by $\left.A\right|_{S} x=A x$ for all $x \in D\left(\left.A\right|_{S}\right):=D(A) \cap S$. For an open set $\Omega \subset \mathbb{C}, H^{\infty}(\Omega, \mathcal{B}(X, Y))$ denotes the set of all bounded analytic $\mathcal{B}(X, Y)$-valued functions $f$ defined on an open set $\Omega_{f} \subset \Omega$ (depending on $f$ ) such that $\Omega \backslash \Omega_{f}$ is a discrete set 1 Clearly, each function $f \in H^{\infty}(\Omega, \mathcal{B}(X, Y))$ admits an extension $\tilde{f} \in H^{\infty}(\Omega, \mathcal{B}(X, Y))$ defined on $\Omega$. For an arbitrary set $\Lambda \subset \mathbb{C}$, we define

$$
H^{\infty}(\Lambda, \mathcal{B}(X, Y)):=\bigcup_{\Omega \supset \Lambda, \Omega \text { open }} H^{\infty}(\Omega, \mathcal{B}(X, Y)) .
$$

\section{THE SAMPLED-DATA SYSTEM}

We start with a simple lemma which will be useful in the subsequent developments.

Lemma 2.1. Let $X, Y$ and $Z$ be Hilbert spaces and let $L:[0,1] \rightarrow \mathcal{B}(X, Y)$ be given. If $\lim _{t \rightarrow 0} L(t) x=0$ for all $x \in X$ and if $K \in \mathcal{B}(Z, X)$ is compact, then

$$
\lim _{t \rightarrow 0}\|L(t) K\|_{\mathcal{B}(Z, Y)}=0 .
$$

Moreover, if $\lim _{t \rightarrow 0} L^{*}(t) y=0$ for all $y \in Y$, and if $H \in \mathcal{B}(Y, Z)$ is compact, then

$$
\lim _{t \rightarrow 0}\|H L(t)\|_{\mathcal{B}(X, Z)}=0 .
$$

Proof. Assume that $\lim _{t \rightarrow 0} L(t) x=0$ for all $x \in X$. If $K \in \mathcal{B}(Z, X)$ has finite rank, then it is easy to prove that (2.1) holds. In a Hilbert space a compact operator can be uniformly approximated by finite rank operators, and a standard argument shows that (2.1) is true for compact $K \in \mathcal{B}(Z, X)$.

Assuming that $\lim _{t \rightarrow 0} L^{*}(t) x=0$ for all $x \in X$ and that $H \in \mathcal{B}(Y, Z)$ is compact, (2.2) can be obtained by an application of (2.1) to $L^{*}(t)$ and $H^{*}$.

Throughout the paper, $X$ and $U$ denote Hilbert spaces. Let us consider the following sampled-data system with state space $X$ and input space $U$ :

$$
\begin{aligned}
& \dot{x}(t)=A x(t)+B u(t), t \geq 0 ; x(0)=x^{0} \in X, \\
& u(t)=F x(k \tau), k \tau \leq t<(k+1) \tau, k \in \mathbb{N}_{0},
\end{aligned}
$$

where $x(t) \in X, u(t) \in U, \tau>0$ is the sampling time, $A$ is the generator of a strongly continuous semigroup $T(t)$ on $X, F \in \mathcal{B}(X, U)$ and $B \in \mathcal{B}(U, X-1)$, where $X_{-1}$ denotes the closure of $X$ in the norm $\|x\|_{-1}=\left\|(\lambda I-A)^{-1} x\right\|$ (here $\|\cdot\|$ denotes the norm of $X$ and $\lambda$ is any element in $\varrho(A))$. Clearly, $X \hookrightarrow X_{-1}$, and hence $\mathcal{B}(U, X) \subset \mathcal{B}\left(U, X_{-1}\right)$. We say that $B$ is bounded if $B \in \mathcal{B}(U, X)$; otherwise we say that $B$ is unbounded. The semigroup $T(t)$ extends to a strongly continuous semigroup on $X_{-1}$. The generator of $T(t)$ on $X_{-1}$ is an extension of $A$ to $X$

\footnotetext{
${ }^{1}$ This means that either $\Omega_{f}=\Omega$ or, if $\Omega_{f} \neq \Omega$, then for each $z \in \Omega \backslash \Omega_{f}$ there exists $\varepsilon>0$ such that $\mathbb{B}(z, \varepsilon) \cap\left(\Omega \backslash \Omega_{f}\right)=\{z\}$.
} 
(which is bounded as an operator from $X$ to $X_{-1}$ ). We shall use the same symbol $T(t)$ (respectively, $A$ ) for the original semigroup (respectively, its generator) and the associated extensions. With this convention, we may write $A \in \mathcal{B}\left(X, X_{-1}\right)$. Considered as a generator on $X_{-1}$, the domain of $A$ is $X$. The spectrum of $A$ considered as an operator on $X$ coincides with the spectrum of $A$ considered as an operator on $X_{-1}$; moreover, the point spectra of $A$ and its extension coincide, including algebraic multiplicities of isolated eigenvalues, We refer the reader to $\mathrm{p}$. 123 in the book [5] by Engel and Nagel for more details on the extrapolation space $X_{-1}$.

The derivative on the left-hand side of (2.3a) has to be interpreted in the space $X_{-1}$. To solve the initial-value problem (2.3), we define a function $x$ recursively by

$$
\begin{aligned}
x(0) & =x^{0}, \\
x(k \tau+t) & =T(t) x(k \tau)+\int_{0}^{t} T(s) B F x(k \tau) d s, \forall t \in(0, \tau], \forall k \in \mathbb{N}_{0} .
\end{aligned}
$$

It follows from standard results in the theory of strongly continuous semigroups (applied to $T(t)$ considered as a semigroup on $X_{-1}$ ) that

$$
x \in C\left(\mathbb{R}_{+}, X\right) \quad \text { and }\left.\quad x\right|_{[k \tau,(k+1) \tau]} \in C^{1}\left([k \tau,(k+1) \tau], X_{-1}\right), \forall k \in \mathbb{N}_{0} .
$$

Moreover, $x$ satisfies the following differential equations in $X_{-1}$ :

$$
\dot{x}(t)=A x(t)+B F x(k \tau), \quad \forall t \in(k \tau,(k+1) \tau), \forall k \in \mathbb{N}_{0} .
$$

It is also clear that $x$ given by (2.4) is the only function with these properties. In this sense, the function $x$ defined by (2.4) is the unique solution of (2.3).

We say that (2.3) is exponentially stable if there exist $N \geq 1$ and $\nu>0$ such that $\|x(t)\| \leq N e^{-\nu t}\left\|x^{0}\right\|$ for all initial values $x^{0} \in X$ and all $t \geq 0$. To study the stability properties of the sampled-data system (2.3) we consider a related discrete time system. Let $x$ be the solution of (2.3) given by (2.4) and set

$$
x_{k}:=x(k \tau) .
$$

Then, by $(2.4 b)$,

$$
x_{k+1}=T(\tau) x_{k}+\int_{0}^{\tau} T(s) B F x_{k} d s=\left(T(\tau)+S_{\tau} F\right) x_{k}, \forall k \in \mathbb{N}_{0},
$$

where $S_{\tau}$ is an operator defined on $U$ and given by

$$
S_{\tau} u:=\int_{0}^{\tau} T(s) B u d s, \quad \forall u \in U .
$$

Lemma 2.2. For any $\tau \geq 0, S_{\tau} \in \mathcal{B}(U, X)$, and for any $\theta>0$,

$$
\sup _{0 \leq \tau \leq \theta}\left\|S_{\tau}\right\|_{\mathcal{B}(U, X)}<\infty .
$$

Moreover, if $F \in \mathcal{B}(X, U)$ is compact, then

$$
\lim _{\tau \rightarrow 0}\left\|S_{\tau} F\right\|_{\mathcal{B}(X)}=0 .
$$

Proof. By standard results from semigroup theory applied to $T(t)$ considered as a semigroup on $X_{-1}$, we know that $S_{\tau} U \subset X$ and

$$
A S_{\tau} u=(T(\tau)-I) B u, \quad \forall u \in U, \forall \tau \in(0, \infty) .
$$


Hence, for $\lambda \in \varrho(A)$, we obtain

$$
\begin{aligned}
S_{\tau} u= & (I-T(\tau))(\lambda I-A)^{-1} B u \\
& +\lambda \int_{0}^{\tau} T(s)(\lambda I-A)^{-1} B u d s, \quad \forall u \in U, \forall \tau \in[0, \infty),
\end{aligned}
$$

showing that $S_{\tau} \in \mathcal{B}(U, X)$ and that (2.7) holds. Moreover, let $F \in \mathcal{B}(X, U)$ be compact. We see from (2.9) that $\lim _{\tau \rightarrow 0}\left\|S_{\tau} u\right\|=0$ for all $u \in U$, and thus (2.8) follows from an application of Lemma 2.1 .

Introducing the operator

$$
\Delta_{\tau}:=T(\tau)+S_{\tau} F,
$$

(2.5) can be written in the form

$$
x_{k+1}=\Delta_{\tau} x_{k}, \forall k \in \mathbb{N} .
$$

Note that by Lemma 2.2 $\Delta_{\tau} \in \mathcal{B}(X)$. Recall that an operator $L \in \mathcal{B}(X)$ is said to be power stable if $\left\|L^{k}\right\| \rightarrow 0$ as $k \rightarrow \infty$.

The following simple result can be obtained by a slight modification of the proof of Proposition 2.1 in Rebarber and Townley [16].

Lemma 2.3. For any $\tau>0$, (2.3) is exponentially stable if and only if $\Delta_{\tau}$ is power stable.

Hence, for the remainder of this paper, we study the power stability of $\Delta_{\tau}$ in order to study the exponential stability of (2.3).

\section{BOUNDED CONTROL}

In this section we assume that the control operator $B$ is bounded. We show that if the feedback operator $F$ is compact, then exponential stability of the continuoustime semigroup generated by $A+B F$ implies exponential stability of the sampleddata system (2.3) provided the sampling time $\tau$ is small enough. More precisely, we prove the following result.

Theorem 3.1. Assume that $A$ generates a strongly continuous semigroup $T(t)$ on $X, B \in \mathcal{B}(U, X), F \in \mathcal{B}(X, U)$ is compact and the semigroup generated by $A+B F$ is exponentially stable. Then there exists $\tau^{*}>0$ such that for every $\tau \in\left(0, \tau^{*}\right)$, there exist $N \geq 1$ and $\nu>0$ such that all solutions of (2.3) satisfy $\|x(t)\| \leq N e^{-\nu t}\left\|x^{0}\right\|$ for all $x^{0} \in X$ and all $t \geq 0$.

Proof. Denote the semigroup generated by $A+B F$ by $T_{B F}(t)$. We start by writing $\Delta_{\tau}$ as a perturbation of $T_{B F}(\tau)$. By the variation of parameters formula from the perturbation theory of semigroups (see, for example, [14, p. 79), we have

$$
T_{B F}(\tau) x=T(\tau) x+\int_{0}^{\tau} T(\tau-s) B F T_{B F}(s) x d s, \quad \forall x \in X .
$$

Using (2.10) and defining $P_{\tau} \in \mathcal{B}(X)$ by

$$
P_{\tau} x:=\int_{0}^{\tau} T(\tau-s) B F\left(I-T_{B F}(s)\right) x d s, \quad \forall x \in X,
$$

we see that

$$
\Delta_{\tau}=T_{B F}(\tau)+P_{\tau} .
$$


By the hypothesis that $T_{B F}$ is exponentially stable, there exist $M \geq 1$ and $\omega>0$ such that

$$
\left\|T_{B F}(t) x\right\| \leq M e^{-\omega t}\|x\|, \forall x \in X, \forall t \in \mathbb{R}_{+} .
$$

Adopting a standard technique from semigroup theory (see, for example, the proof of Theorem 5.2 on p. 19 in Pazy [14]), we introduce a new norm $|\cdot|$ on $X$ by setting

$$
|x|:=\sup _{t \geq 0}\left\|e^{\omega t} T_{B F}(t) x\right\|, \quad \forall x \in X .
$$

Clearly,

$$
\|x\| \leq|x| \leq M\|x\|, \quad \forall x \in X
$$

and, moreover,

$$
\begin{aligned}
\left|T_{B F}(t) x\right| & =\sup _{s \geq 0}\left\|e^{\omega s} T_{B F}(s) T_{B F}(t) x\right\| \leq e^{-\omega t} \sup _{s \geq 0}\left\|e^{\omega s} T_{B F}(s) x\right\| \\
& =e^{-\omega t}|x|, \quad \forall x \in X, \forall t \geq 0 .
\end{aligned}
$$

Since $F$ is compact and $\lim _{s \rightarrow 0}\left(I-T_{B F}(s)\right) x=0$ for all $x \in X$, an application of Lemma 2.1 yields

$$
\lim _{s \rightarrow 0}\left\|F\left(I-T_{B F}(s)\right)\right\|_{\mathcal{B}(X, U)}=0 .
$$

For $L \in \mathcal{B}(X)$ we denote the operator norm $\sup _{x \in X}(|L x| /|x|)$ by $|L|$. Using (3.1)(3.3) and invoking the inequality

$$
e^{-\omega \tau} \leq 1-\omega \tau e^{-\omega \tau}, \quad \forall \tau \in \mathbb{R}_{+},
$$

we obtain

$$
\begin{aligned}
\left|\Delta_{\tau}\right| & \leq e^{-\omega \tau}+\left|P_{\tau}\right| \leq e^{-\omega \tau}+M\left\|P_{\tau}\right\| \\
& \leq 1-\omega \tau+\left[\omega\left(1-e^{-\omega \tau}\right)+h(\tau)\right] \tau, \forall \tau \in \mathbb{R}_{+},
\end{aligned}
$$

where

$$
h(\tau):=M \sup _{0 \leq s \leq \tau}\left\|T(\tau-s) B F\left(I-T_{B F}(s)\right)\right\|_{\mathcal{B}(X)} .
$$

By (3.4),

$$
\lim _{\tau \rightarrow 0^{+}} h(\tau)=0,
$$

and hence it follows from (3.5) that for fixed but arbitrary $\varepsilon \in(0, \omega)$ there exists $\tau_{\varepsilon}>0$ such that

$$
\left|\Delta_{\tau}\right|<1-(\omega-\varepsilon) \tau<1, \forall \tau \in\left(0, \tau_{\varepsilon}\right) .
$$

Applying (3.2), we see that for any $\tau \in\left(0, \tau_{\varepsilon}\right)$,

$$
\left\|\Delta_{\tau}^{k} x\right\| \leq\left|\Delta_{\tau}^{k} x\right| \leq\left|\Delta_{\tau}^{k}\right||x| \leq M\left|\Delta_{\tau}\right|^{k}\|x\|, \quad \forall x \in X, \forall k \in \mathbb{N} .
$$

Combining this with (3.6) shows that $\Delta_{\tau}$ is power stable for all $\tau \in\left(0, \tau_{\varepsilon}\right)$.

Remark 3.2. (1) Examining the proof of Theorem 3.1, we see that for every $\varepsilon \in$ $(0, \omega)$, there exists $\tau_{\varepsilon}>0$ such that

$$
\left\|\Delta_{\tau}^{k}\right\| \leq M(1-(\omega-\varepsilon) \tau)^{k}, \forall k \in \mathbb{N}, \forall \tau \in\left(0, \tau_{\varepsilon}\right) .
$$

(2) A key element in the proof of Theorem 3.1 is that $\lim _{\tau \rightarrow 0^{+}} h(\tau)=0$, which implies that

$$
\lim _{\tau \rightarrow 0^{+}} \frac{\left\|P_{\tau}\right\|}{\tau}=0
$$


In the case of unbounded $B$, this does not seem to be true, even if $B$ is an admissible control operator for $T(t)$ (in the sense of Weiss [21]).

The next remark shows that the compactness assumption on $F$ imposed in Theorem 3.1 cannot be relaxed.

Remark 3.3. It is easy to find counterexamples which show that, in general, Theorem 3.1 is not true for non-compact feedback operators $F$. Indeed, consider the case where $X=l^{2}(\mathbb{Z}), A=\operatorname{diag}_{k \in \mathbb{Z}}(1+k i), B=I$ and $F=-2 I$. Then $F$ is a continuous-time exponentially stabilizing feedback, since $A+B F$ generates the exponentially stable semigroup

$$
T_{B F}(t)=\operatorname{diag}_{k \in \mathbb{Z}}\left(e^{(-1+k i) t}\right) .
$$

However, applying the sampled-data feedback given by (2.3b) results in the discretetime operator

$$
\Delta_{\tau}=\operatorname{diag}_{k \in \mathbb{Z}}\left(e^{(1+k i) \tau}\right)-2 \operatorname{diag}_{k \in \mathbb{Z}}\left(\frac{e^{(1+k i) \tau}-1}{1+k i}\right) .
$$

Since

$$
\left|e^{(1+k i) \tau}-2\left(\frac{e^{(1+k i) \tau}-1}{1+k i}\right)\right| \geq e^{\tau}-\frac{2}{|1+i k|}\left(e^{\tau}+1\right),
$$

we see that for any $\tau>0$, the eigenvalues $\lambda_{k}^{\tau}$ of $\Delta_{\tau}$ satisfy

$$
\liminf _{|k| \rightarrow \infty}\left|\lambda_{k}^{\tau}\right| \geq e^{\tau}>1,
$$

showing that $\Delta_{\tau}$ is not power stable for any $\tau>0$.

Furthermore, it is possible to find counterexamples which show that, in general, Theorem 3.1 is not true when $B$ is not bounded. In particular, in [17] an example is given for which $U$ is one-dimensional, $F$ is bounded, $B$ is not bounded, $A+B F$ generates an exponentially stable semigroup, $A^{-\varepsilon} B \in \mathcal{B}(U, X)$ for any $\varepsilon>0$ (so $B$ is "barely unbounded" by a common measure of unboundedness), and there exists a sequence of positive sampling times $\left(\tau_{n}\right)$ such that $\tau_{n} \rightarrow 0$ and $\Delta_{\tau_{n}}$ is not power stable.

Remark 3.4. It is important to realise that Theorem 3.1 is completely general in the sense that it applies to any generator $A$, any bounded control operator $B$ and, in particular, any compact stabilizing feedback $F$. Obviously, in specific cases, the result could be proved using different, possibly simpler arguments. As an obvious example, consider the case of a system with a finite-dimensional unstable part (typically arising from a parabolic PDE) and a feedback that stabilizes the unstable part and is zero on the stable invariant subspace. More precisely, in this case there exists a projection operator $\Pi$ on $X$ such that $X_{1}:=\Pi X$ (the unstable subspace) and $X_{2}:=(I-\Pi)(X)$ (the stable subspace) are invariant with respect to the underlying semigroup, $X_{1}$ is finite-dimensional and $X_{1} \subset D(A)$; moreover,

$$
A=\left(\begin{array}{cc}
A_{1} & 0 \\
0 & A_{2}
\end{array}\right), \quad B=\left(\begin{array}{c}
B_{1} \\
B_{2}
\end{array}\right)
$$

where $A_{1}:=\left.A\right|_{X_{1}}, A_{2}:=\left.A\right|_{X_{2}}, B_{1}:=\Pi B, B_{2}:=(I-\Pi) B$ and the operator $A_{2}$ generates an exponentially stable semigroup on $X_{2}$ (see, for example, Lemma 2.5.7 in Curtain and Zwart [3] for more details). If $F_{1}: X_{1} \rightarrow U$ is an exponentially stabilizing feedback for the (finite-dimensional) system $\left(A_{1}, B_{1}\right)$, then $F:=\left(F_{1}, 0\right)$ 
is an exponentially stabilizing feedback for $(A, B)$. It is obvious from the wellknown finite-dimensional version of Theorem 3.1 that for this particular feedback the corresponding sampled-data feedback system (2.3) is exponentially stable for all suffciently small $\tau>0$. Clearly, one could imagine proofs tailored to other situations with specific feedbacks, say for example hyperbolic PDEs, retarded and neutral functional equations or mixed parabolic/hyperbolic systems. However, in many significant applications there is little scope for actually choosing the feedback. For example, in static output feedback, the basic structure of the feedback is inherited from the given observation and the feedback design is limited to choosing feedback gains. In such cases it is difficult to see the merits of specifically tailored proofs or indeed how they would be developed. It is therefore an important aspect of Theorem 3.1 that it does not make any assumptions on the specific structure of the underlying system $(A, B)$ and the stabilizing feedback $F$.

In the next section we show that Theorem 3.1 generalises to the case with unbounded $B$ in the special, but important, case where $A$ generates an analytic semigroup.

\section{UnBOUNDED CONTROL AND ANALYTIC SEMIGROUP}

In order to formulate and prove the main result of this section a number of preparations are required. Throughout this section, we assume that $A$ generates a strongly continuous semigroup $T(t)$ on $X, B \in \mathcal{B}\left(U, X_{-1}\right)$ and $F \in \mathcal{B}(X, U)$. Further assumptions on $(A, B, F)$ will be introduced when needed. We define an operator $A_{B F}$ on $X$ by setting

$$
A_{B F} x=(A+B F) x, \quad \forall x \in D\left(A_{B F}\right):=\{x \in X \mid(A+B F) x \in X\} .
$$

It is easy to show that $A_{B F}$ is closed. We carefully distinguish between the operators $A_{B F}$ and $A+B F$, the latter being an unbounded operator on $X_{-1}$ with domain $D(A+B F)=X$. Clearly, $A+B F \in \mathcal{B}\left(X, X_{-1}\right)$. Note that $A_{B F}$ is the part of $A+B F$ in $X$. The transfer function of the continuous-time system given by $(A, B, F)$ is defined as follows:

$$
\mathbf{G}: \varrho(A) \rightarrow \mathcal{B}(U), \quad s \mapsto F(s I-A)^{-1} B .
$$

We introduce the space $X_{*}:=D\left(A^{*}\right)$, endowed with the norm

$$
\|x\|_{*}:=\left\|\left(\bar{\lambda} I-A^{*}\right) x\right\|,
$$

where $\lambda$ is any element of $\varrho(A)$. Clearly, $X_{1} \hookrightarrow X=X^{*}$. It is well known that $X_{-1}$ and $\left(X_{*}\right)^{*}$ are isometrically isomorphic. Therefore, the triple $X_{*} \hookrightarrow X=X^{*} \hookrightarrow$ $X_{-1}$ is a so-called Gelfand triple. Moreover, if $B \in \mathcal{B}\left(U, X_{-1}\right)$, then $B^{*} \in \mathcal{B}\left(X_{*}, U\right)$.

Lemma 4.1. Under the above assumptions on $A, B$ and $F$, the following statements hold:

(1) $\varrho(A+B F) \cap \varrho(A)=\{s \in \varrho(A) \mid 1 \in \varrho(\mathbf{G}(s))\}$;

(2) if $\varrho(A+B F) \neq \emptyset$, then $\varrho\left(A_{B F}\right)=\varrho(A+B F)$ and

$$
\left(s I-A_{B F}\right)^{-1}=\left.(s I-A-B F)^{-1}\right|_{X}, \quad \forall s \in \varrho\left(A_{B F}\right)=\varrho(A+B F) ;
$$

(3) if $F$ is compact, then

$$
\varrho\left(A_{B F}\right) \cap \varrho(A) \subset \varrho(A+B F) ;
$$

(4) if $F$ is compact, then $A_{B F}^{*}=A^{*}+F^{*} B^{*}$ with $D\left(A_{B F}^{*}\right)=D\left(A^{*}\right)=X_{*}$. 
Proof. To prove statements (1) and (3), let $s \in \varrho(A)$, and write

$$
s I-A-B F=(s I-A)\left[I-(s I-A)^{-1} B F\right] .
$$

It follows that

$$
s \in \varrho(A+B F) \Leftrightarrow 1 \in \varrho\left((s I-A)^{-1} B F\right) \Leftrightarrow 1 \in \varrho\left(F(s I-A)^{-1} B\right),
$$

yielding statement (1). Assume that $F$ is compact. Then, by (4.3),

$$
s \in \sigma(A+B F) \Leftrightarrow 1 \in \sigma_{p}\left((s I-A)^{-1} B F\right) .
$$

Therefore, if $s \in \sigma(A+B F)$, there exists $x \in X, x \neq 0$, such that $(s I-A)^{-1} B F x=$ $x$. Hence, $(A+B F) x=s x$, showing that $s \in \sigma_{p}\left(A_{B F}\right)$. We may conclude that $\sigma(A+B F) \cap \varrho(A) \subset \sigma\left(A_{B F}\right)$, proving statement (3).

To prove statement $(2)$, let $\lambda \in \varrho(A+B F)$ and note that, by Proposition 2.17 on p. 261 in [5], we only need to show that the topology on $X$ given by the norm

$$
x \mapsto|x|=\|(\lambda I-A-B F) x\|_{-1},
$$

is stronger than the original norm topology. But this follows immediately, since with

$$
k:=\left\|(\lambda I-A-B F)^{-1}\right\|_{\mathcal{B}\left(X_{-1}, X\right)}, \quad l:=\|\lambda I-A-B F\|_{\mathcal{B}\left(X, X_{-1}\right)} .
$$

we have

$$
\|x\| \leq k\|(\lambda I-A-B F) x\|_{-1}=k|x|, \quad|x| \leq l\|x\| ; \quad \forall x \in X,
$$

i.e., the two norms are even equivalent.

To prove statement (4), consider the operator $A^{*}+F^{*} B^{*}$ with $D\left(A^{*}+F^{*} B^{*}\right)=$ $D\left(A^{*}\right)=X_{*}$. The compactness of $F$ together with the fact that $B^{*} \in \mathcal{B}\left(X_{*}, U\right)$ yields that $F^{*} B^{*}$ is relatively compact with respect to $A^{*}$. Therefore, since $A^{*}$ is closed, $A^{*}+F^{*} B^{*}$ is closed (see Theorem 1.1 on p. 194 in [8]) and so

$$
\left(A^{*}+F^{*} B^{*}\right)^{* *}=A^{*}+F^{*} B^{*}
$$

(see Theorem 5.29 on p. 168 in [8]). As a consequence, statement (4) will follow if we can show that

$$
\left(A^{*}+F^{*} B^{*}\right)^{*}=A_{B F} .
$$

To this end assume that $x \in D\left(\left(A^{*}+F^{*} B^{*}\right)^{*}\right)$. Setting $x^{\prime}:=\left(A^{*}+F^{*} B^{*}\right)^{*} x \in X$, we have that

$$
\left\langle x,\left(A^{*}+F^{*} B^{*}\right) z\right\rangle=\left\langle x^{\prime}, z\right\rangle, \quad \forall z \in X_{*},
$$

where $\langle\cdot, \cdot\rangle$ denotes the inner product in $X$. The duality pairing on $X_{-1} \times X_{*}$, denoted by $[\cdot, \cdot]$, is given by

$$
[y, z]:=\left\langle(\lambda I-A)^{-1} y,\left(\bar{\lambda} I-A^{*}\right) z\right\rangle, \quad \forall(y, z) \in X_{-1} \times X_{*},
$$

where $\lambda \in \varrho(A)$. It is straightforward to show that

$[y, z]=\langle y, z\rangle, \quad[A y, z]=\left\langle y, A^{*} z\right\rangle, \quad[B F y, z]=\left\langle y, F^{*} B^{*} z\right\rangle ; \quad \forall(y, z) \in X \times X_{*}$.

Thus, by (4.4),

$$
\left[x^{\prime}-(A+B F) x, z\right]=0, \quad \forall z \in X_{*},
$$

and so $(A+B F) x=x^{\prime} \in X$, proving that $x \in D\left(A_{B F}\right)$ and $A_{B F} x=\left(A^{*}+F^{*} B^{*}\right)^{*} x$. This shows that $A_{B F}$ is an extension of $\left(A^{*}+F^{*} B^{*}\right)^{*}$. It remains to prove that 
$D\left(A_{B F}\right) \subset D\left(\left(A^{*}+F^{*} B^{*}\right)^{*}\right)$. Let $x \in D\left(A_{B F}\right)$. Invoking (4.5), we obtain for all $z \in X_{*}$,

$$
\left\langle x,\left(A^{*}+F^{*} B^{*}\right) z\right\rangle=[(A+B F) x, z]=\left\langle A_{B F} x, z\right\rangle,
$$

showing that $x \in D\left(\left(A^{*}+F^{*} B^{*}\right)^{*}\right)$.

Remark 4.2. Statements (2) and (3) of Lemma 4.1 are wrong if the operator $A_{B F}$ is replaced by a proper restriction of $A_{B F}$. More precisely, consider an operator $\tilde{A}_{B F}$ on $X$ given by

$$
\tilde{A}_{B F} x=(A+B F) x, \quad \forall x \in D\left(\tilde{A}_{B F}\right) \subset D\left(A_{B F}\right) .
$$

If $D\left(\tilde{A}_{B F}\right) \neq D\left(A_{B F}\right)$, then

$$
\varrho\left(\tilde{A}_{B F}\right) \subset \sigma_{p}\left(A_{B F}\right) \subset \sigma_{p}(A+B F) .
$$

The second inclusion holds trivially. To prove the first inclusion, let $\lambda \in \varrho\left(\tilde{A}_{B F}\right)$. Then, $\lambda I-\tilde{A}_{B F}: D\left(\tilde{A}_{B F}\right) \rightarrow X$ is bijective and hence, for $y \in D\left(A_{B F}\right) \backslash D\left(\tilde{A}_{B F}\right)$, there exists $x \in D\left(\tilde{A}_{B F}\right)$ such that $\left(\lambda I-\tilde{A}_{B F}\right) x=\left(\lambda I-A_{B F}\right) y$. It follows that $\left(\lambda I-A_{B F}\right)(y-x)=0$, and since $y-x \neq 0$, we see that $\lambda \in \sigma_{p}\left(A_{B F}\right)$.

Moreover, combining statement (1) of Lemma 4.1 and (4.6), we see that if $\tilde{A}_{B F}$ is a proper restriction of $A_{B F}$, then $I-\mathbf{G}(s)$ is not invertible for any $s \in \varrho\left(\tilde{A}_{B F}\right) \cap$ $\varrho(A)$.

Lemma 4.3. Assume that $F \in \mathcal{B}(X, U)$ is compact and that the following two spectral assumptions hold:

(S1) $\overline{\mathbb{C}}_{0} \subset \varrho\left(A_{B F}\right)$;

(S2) there exists $\varepsilon>0$ such that $\sigma(A) \cap \mathbb{C}_{-\varepsilon}$ is bounded.

Then there exists $\alpha<0$ such that $\sigma(A) \cap \mathbb{C}_{\alpha}$ consists of finitely many eigenvalues of $A$ with finite algebraic multiplicities.

Proof. Since $F$ is compact, it follows from statement (3) of Lemma 4.1 and assumption (S1) that $\varrho(A+B F) \neq \emptyset$. Hence, combining statement (2) of Lemma 4.1, assumption (S1) and the fact that $\varrho\left(A_{B F}\right)$ is open shows that there exists an open set $\Omega$ such that

$$
\varrho(A+B F)=\varrho\left(A_{B F}\right) \supset \Omega \supset \overline{\mathbb{C}}_{0} .
$$

Recall that the spectrum of $A$ considered as an operator on $X$ coincides with the spectrum of $A$ considered as an operator on $X_{-1}$ (see [5, p. 261, Proposition 2.17). Moreover, the point spectra of $A$ and its extension are identical, including algebraic multiplicities of isolated eigenvalues, For the rest of this proof we will consider $A$ as an operator on $X_{-1}$ with domain $X$. Clearly,

$$
s I-A=\left[I+B F(s I-A-B F)^{-1}\right](s I-A-B F), \quad \forall s \in \Omega,
$$

and therefore, by the compactness of $F$,

$$
s \in \sigma(A) \Leftrightarrow-1 \in \sigma_{p}\left(B F(s I-A-B F)^{-1}\right), \quad \forall s \in \Omega .
$$

Let $s \in \sigma(A) \cap \Omega$. Then, by (4.8) and (4.9), there exists $x \in X_{-1}, x \neq 0$, such that

$$
(s I-A)(s I-A-B F)^{-1} x=0,
$$

showing that $s$ is an eigenvalue of $A$, and so

$$
\sigma(A) \cap \Omega=\sigma_{p}(A) \cap \Omega .
$$


Combining (4.9), (4.10) and the compactness of $F$ with Theorem 1.9 on p. 370 in Kato 8 ] shows that for any compact set $K \subset \Omega$, the intersection $\sigma_{p}(A) \cap K$ is finite. It now follows from assumption (S2) that there exists $\alpha<0$ such that $\sigma_{p}(A) \cap \mathbb{C}_{\alpha}$ is finite and $\sigma_{p}(A) \cap \mathbb{C}_{\alpha} \subset \Omega$. It remains to show that each element in $\sigma_{p}(A) \cap \mathbb{C}_{\alpha}$ has finite algebraic multiplicity. Seeking a contradiction, let $\lambda \in \sigma_{p}(A) \cap \mathbb{C}_{\alpha} \subset \Omega$ and suppose that $\lambda$ has infinite algebraic multiplicity. Then, by Theorem 5.28 on p. 239 in [8], $\lambda$ belongs to the essential spectrum of $A$ (as defined on p. 243 in [8]). As an unbounded perturbation on $X_{-1}$, the operator $B F$ is relatively compact with respect to $A$ (considered on $X_{-1}$ ), due to the compactness of $F$. By Theorem 5.35 on p. 244 in [8], the essential spectrum of an operator remains fixed under relatively compact perturbations, and thus, $\lambda \in \sigma(A+B F) \cap \Omega$, contradicting (4.7).

Assume that $F \in \mathcal{B}(X, U)$ is compact and the assumptions (S1) and (S2) hold. Then, by Lemma 4.3, there exists $\alpha<0$ such that $\sigma(A) \cap \mathbb{C}_{\alpha}$ consists of finitely many eigenvalues of $A$ with finite algebraic multiplicities. Hence, there exists a simple closed curve $\gamma$ in the half-plane $\mathbb{C}_{\alpha}$ not intersecting $\sigma(A)$ and enclosing $\sigma(A) \cap \overline{\mathbb{C}}_{0}$ in its interior. The operator

$$
\Pi:=\frac{1}{2 \pi i} \int_{\gamma}(s I-A)^{-1} d s
$$

is a projection operator, and we have

$$
X=X_{1} \oplus X_{2}, \quad \text { where } \quad X_{1}:=\Pi X, \quad X_{2}:=(I-\Pi) X .
$$

By a standard result (see, for example, Lemma 2.5.7 in Curtain and Zwart [3]), the above decomposition has the following properties:

(D1) $\operatorname{dim} X_{1}<\infty$ and $X_{1} \subset D(A)$;

(D2) $X_{1}$ and $X_{2}$ are $T(t)$-invariant for all $t \geq 0$;

(D3) $\sigma\left(\left.A\right|_{X_{1}}\right)=\sigma(A) \cap \overline{\mathbb{C}}_{0}$ and $\sigma\left(\left.A\right|_{X_{2}}\right)=\sigma(A) \cap\left(\mathbb{C} \backslash \overline{\mathbb{C}}_{0}\right)$.

It is useful to introduce the following notation:

$$
A_{j}:=\left.A\right|_{X_{j}}, \quad T_{j}(t):=\left.T(t)\right|_{X_{j}} \quad j=1,2 .
$$

Clearly, by (D1) and (D2), $T_{1}(t)$ is a semigroup on the finite-dimensional space $X_{1}$ with generator $A_{1}$, i.e., $T_{1}(t)=e^{A_{1} t}$, and $T_{2}(t)$ is a strongly continuous semigroup on $X_{2}$ with generator $A_{2}$. Since the spectrum of $A$ considered as an operator on $X$ coincides with the spectrum of $A$ considered as an operator on $X_{-1}$, the projection operator $\Pi$ on $X$ defined in (4.11) extends to a projection on $X_{-1}$. We will use the same symbol $\Pi$ for the original projection and its associated extension. The decomposition (4.12) induces decompositions of the control operator $B \in \mathcal{B}\left(U, X{ }_{-1}\right)$ and the feedback operator $F \in \mathcal{B}(X, U)$ :

$$
B_{1}:=\Pi B, \quad B_{2}:=(I-\Pi) B, \quad F_{1}:=\left.F\right|_{X_{1}}, \quad F_{2}:=\left.F\right|_{X_{2}} .
$$

Lemma 4.4. Assume that $F \in \mathcal{B}(X, U)$ is compact and that assumptions (S1) and (S2) hold. Then the pair $\left(A_{1}, B_{1}\right)$ is controllable and the pair $\left(A_{1}, F_{1}\right)$ is observable.

Proof. Seeking a contradiction, suppose that $\left(A_{1}, B_{1}\right)$ is not controllable. Then, by the Kalman controllability decomposition lemma, we may assume, without loss of generality, that $A_{1}$ and $B_{1}$ can be written in the form

$$
A_{1}=\left(\begin{array}{cc}
A_{11} & * \\
0 & A_{12}
\end{array}\right), \quad B_{1}=\left(\begin{array}{c}
B_{11} \\
0
\end{array}\right) \text {. }
$$


A straightforward calculation combined with an application of statement (2) of Lemma4.1 shows that

$$
\left(s I-A_{B F}\right)^{-1}=(s I-A)^{-1}+(s I-A)^{-1} B F\left(s I-A_{B F}\right)^{-1}, \quad \forall s \in \varrho(A) \cap \overline{\mathbb{C}}_{0} .
$$

Using the decomposition of $A$ and $B$ and (4.15), we see that $(s I-A)^{-1} B F$ may be written in the form

$$
(s I-A)^{-1} B F=\left(\begin{array}{ccc}
\left(s I-A_{11}\right)^{-1} & * & 0 \\
0 & \left(s I-A_{12}\right)^{-1} & 0 \\
0 & 0 & \left(s I-A_{2}\right)^{-1}
\end{array}\right)\left(\begin{array}{ccc}
* & * & * \\
0 & 0 & 0 \\
* & * & *
\end{array}\right) .
$$

Let $\lambda \in \sigma\left(A_{12}\right)$ and let $y$ be a corresponding eigenvector of $A_{12}$. Note that, by (D3), $\lambda \in \overline{\mathbb{C}}_{0}$. Setting $x:=\operatorname{col}(0, y, 0) \in X$, we may conclude from (4.16) and (4.17) that

$$
\left(s I-A_{B F}\right)^{-1} x=\left(\begin{array}{c}
* \\
\left(s I-A_{12}\right)^{-1} y \\
0
\end{array}\right)+\left(\begin{array}{c}
* \\
0 \\
*
\end{array}\right)=\left(\begin{array}{c}
* \\
(s-\lambda)^{-1} y \\
*
\end{array}\right) .
$$

This shows that $\left\|\left(s I-A_{B F}\right)^{-1} x\right\| \rightarrow \infty$ as $s \rightarrow \lambda$ in $\varrho(A) \cap \overline{\mathbb{C}}_{0}$, leading to a contradiction, since by (S1), $\lambda \in \varrho\left(A_{B F}\right)$.

To show the observability of $\left(A_{1}, F_{1}\right)$, note that

$$
\left(s I-A_{B F}\right)^{-1}=(s I-A)^{-1}+(s I-A-B F)^{-1} B F(s I-A)^{-1}, \forall s \in \varrho(A) \cap \overline{\mathbb{C}}_{0},
$$

which can be obtained from a straightforward calculation combined with an application of statement (2) of Lemma 4.1. Based on the Kalman observability decomposition lemma and (4.18), an argument similar to that establishing controllability of $\left(A_{1}, B_{1}\right)$ can be used to prove observability of $\left(A_{1}, F_{1}\right)$. We omit the details for the sake of brevity.

The next result shows that if $A$ generates an analytic semigroup and if $F$ is compact, then $A_{B F}$ generates an analytic semigroup.

Proposition 4.5. Assume that $A$ generates an analytic semigroup $T(t)$ on $X$, $B \in \mathcal{B}\left(U, X_{-1}\right)$, and $F \in \mathcal{B}(X, U)$ is compact. Then $A_{B F}$ generates an analytic semigroup $T_{B F}(t)$ on $X$. Moreover, if $T_{B F}(t)$ is exponentially stable, then ( $I-$ $\mathbf{G})^{-1} \in H^{\infty}\left(\mathbb{C}_{0}, \mathcal{B}(U)\right)$, where $\mathbf{G}$ is given by 4.2 .

Proof. By statement (4) of Lemma 4.1, $A_{B F}^{*}=A^{*}+F^{*} B^{*}$ with $D\left(A_{B F}^{*}\right)=$ $D\left(A^{*}\right)=X_{1}$. Clearly, since $A$ generates an analytic semigroup, so does $A^{*}$. Moreover, by the assumptions on $B$ and $F, B^{*} \in \mathcal{B}\left(X_{1}, U\right)$ and $F^{*}$ is compact, and so $F^{*} B^{*}$ is relatively compact with respect to $A^{*}$. By a well-known result from the perturbation theory of analytic semigroups (see Corollary 2.17 on p. 180 in 5] or Proposition 1 in Zabczyk [23]), it follows that $A_{B F}^{*}=A^{*}+F^{*} B^{*}$ generates an analytic semigroup, and hence, so does $A_{B F}^{* *}$. By the closedness of $A_{B F}$, we have that $A_{B F}^{* *}=A_{B F}$, and thus we may conclude that $A_{B F}$ generates an analytic semigroup $T_{B F}(t)$.

Assume that $T_{B F}(t)$ is exponentially stable. Then, by Lemma 4.1

$$
\varrho\left(A_{B F}\right)=\varrho(A+B F) \supset \overline{\mathbb{C}}_{0},
$$

and for all $s \in \overline{\mathbb{C}}_{0}$ and for all $x \in X_{-1}$ we have that

$$
(s I-A-B F)^{-1} x=(A+B F)(s I-A-B F)^{-1}(A+B F)^{-1} x .
$$


Since $(A+B F)^{-1} x \in X$, we may conclude, using Lemma 4.1 that for all $s \in \overline{\mathbb{C}}_{0}$ and all $x \in X_{-1}$,

$$
\begin{aligned}
(s I-A-B F)^{-1} x & =A_{B F}\left(s I-A_{B F}\right)^{-1}(A+B F)^{-1} x \\
& =\left(s\left(s I-A_{B F}\right)^{-1}-I\right)(A+B F)^{-1} x .
\end{aligned}
$$

A straightforward calculation shows that

$$
(I-\mathbf{G}(s))^{-1}=(I-\mathbf{G}(s))^{-1} \mathbf{G}(s)+I=F(s I-A-B F)^{-1} B+I,
$$

and so, by (4.19),

$$
(I-\mathbf{G}(s))^{-1}=F\left(s\left(s I-A_{B F}\right)^{-1}-I\right)(A+B F)^{-1} B+I .
$$

Since $T_{B F}(t)$ is an exponentially stable analytic semigroup and since $(A+B F)^{-1} B \in$ $\mathcal{B}(U, X)$, the right-hand side of (4.20) is uniformly bounded for $s \in \mathbb{C}_{0}$. Therefore, $(I-\mathbf{G})^{-1} \in H^{\infty}\left(\mathbb{C}_{0}, \mathcal{B}(U)\right)$.

Corollary 4.6. Assume that $A$ generates an analytic semigroup $T(t)$ on $X, B \in$ $\mathcal{B}\left(U, X_{-1}\right)$, and $F \in \mathcal{B}(X, U)$ is compact. If the semigroup generated by $A_{B F}$ is exponentially stable, then there exists a decomposition $X=X_{1} \oplus X_{2}$ such that (D1)-(D3) are satisfied and, moreover, the following property holds:

(D4) $T_{2}(t)$ is an exponentially stable analytic semigroup on $X_{2}$ with generator $A_{2}$, where $T_{2}(t)$ and $A_{2}$ are given by (4.13).

Proof. By the exponential stability of the semigroup generated by $A_{B F}$, assumption (S1) in Lemma 4.3 holds. Since $A$ generates an analytic semigroup, for any $\beta \in \mathbb{R}$, the intersection $\sigma(A) \cap \mathbb{C}_{\beta}$ is bounded, and so assumption (S2) in Lemma 4.3 is satisfied. Therefore, by Lemma 4.3, properties (D1)-(D3) hold. Moreover, it is clear that the semigroup $T_{2}(t)$ is analytic, and therefore satisfies the spectrum determined growth assumption. By (D3) and Lemma 4.3, the generator $A_{2}$ of $T_{2}(t)$ satisfies $\sigma\left(A_{2}\right) \subset \mathbb{C} \backslash \mathbb{C}_{-\varepsilon}$ for some $\varepsilon>0$. Therefore, we may conclude that $T_{2}(t)$ is exponentially stable.

Consider the discrete-time system $\left(T(\tau), S_{\tau}, F\right)$, where $S_{\tau}$ is given by (2.6), and denote the transfer function of this system by $\mathbf{H}_{\tau}$, i.e.,

$$
\mathbf{H}_{\tau}(z)=F(z I-T(\tau))^{-1} S_{\tau} .
$$

Then, under the assumptions of Lemma 4.3 (i.e., $F$ is compact and (S1) and (S2) hold), we may write

$$
\mathbf{H}_{\tau}=\mathbf{H}_{\tau}^{1}+\mathbf{H}_{\tau}^{2},
$$

where, using the notation of (4.13) and (4.14),

$$
\mathbf{H}_{\tau}^{j}(z):=F_{j}\left(z I-T_{j}(\tau)\right)^{-1} \int_{0}^{\tau} T_{j}(s) B_{j} d s, \quad j=1,2 .
$$

In light of Lemma 2.3 in order to prove that the sampled-data system (2.3) is exponentially stable for a given sampling time $\tau>0$, we need to show that $\Delta_{\tau}$ given by (2.10) is power stable. The following lemma provides a sufficient condition for the power stability of $\Delta_{\tau}$ in terms of $\tau$ and $\mathbf{H}_{\tau}$.

Lemma 4.7. Assume that $A$ generates an analytic semigroup $T(t)$ on $X, B \in$ $\mathcal{B}(U, X-1), F \in \mathcal{B}(X, U)$ is compact and the semigroup generated by $A_{B F}$ is exponentially stable. If $\tau>0$ is such that

$$
\tau\left(\lambda_{2}-\lambda_{1}\right) \neq 2 k \pi i, \quad \forall k \in \mathbb{Z} \backslash\{0\}, \forall \lambda_{1}, \lambda_{2} \in \sigma(A) \cap \overline{\mathbb{C}}_{0},
$$


and if $\left(I-\mathbf{H}_{\tau}\right)^{-1} \in H^{\infty}(\mathbb{E}, \mathcal{B}(U))$, then $\Delta_{\tau}$ is power stable.

Note that under the assumptions of the above lemma, (4.24) is satisfied for all sufficiently small $\tau>0$.

Proof of Lemma 4.7. By Corollary 4.6 there exists a decomposition $X=X_{1} \oplus X_{2}$ such that (D1)-(D4) hold. In particular, using the notation of (4.13) and (4.14), we have $\sigma\left(A_{1}\right)=\sigma(A) \cap \overline{\mathbb{C}}_{0}$, and, moreover, setting $S_{\tau}^{j}:=\int_{0}^{\tau} T_{j}(s) B_{j} d s, j=1,2$, we may write

$$
T(\tau)=\left(\begin{array}{cc}
T_{1}(\tau) & 0 \\
0 & T_{2}(\tau)
\end{array}\right), \quad S_{\tau}=\left(\begin{array}{c}
S_{\tau}^{1} \\
S_{\tau}^{2}
\end{array}\right), \quad F=\left(F_{1}, F_{2}\right) .
$$

Assume that $\tau$ satisfies (4.24). By Lemma 4.4 the finite-dimensional controlled system $\left(A_{1}, B_{1}\right)$ is controllable, and so, by a well-known result (see 7] or Theorem 4 on p. 102 in Sontag [19]), the discrete-time system $\left(T_{1}(\tau), S_{\tau}^{1}\right)$ is controllable as well. This implies, in particular, that there exists $K_{1} \in \mathcal{B}\left(X_{1}, U\right)$ such that the matrix $T_{1}(\tau)+S_{\tau}^{1} K_{1}$ is power stable. Now $T_{2}(\tau)$ is power stable (since the semigroup $T_{2}(t)$ is exponentially stable by (D4)) and, setting $K:=\left(K_{1}, 0\right) \in \mathcal{B}(X, U)$, it follows from (4.25) that $T(\tau)+S_{\tau} K$ is power stable, showing that the controlled discretetime system $\left(T(\tau), S_{\tau}\right)$ is stabilizable. A similar argument shows that the observed discrete-time system $(T(\tau), F)$ is detectable. Hence, if $\left(I-\mathbf{H}_{\tau}\right)^{-1} \in H^{\infty}(\mathbb{E}, \mathcal{B}(U))$, we may conclude that $\Delta_{\tau}$ is power stable (see Theorem 2 in Logemann [9]).

The following theorem is the main result of this section.

Theorem 4.8. Assume that $A$ generates an analytic semigroup $T(t)$ on $X, B \in$ $\mathcal{B}\left(U, X_{-1}\right)$, and $F \in \mathcal{B}(X, U)$ is compact. If the semigroup generated by $A_{B F}$ is exponentially stable, then there exists $\tau^{*}>0$ such that for every $\tau \in\left(0, \tau^{*}\right)$, there exist $N \geq 1$ and $\nu>0$ such that all solutions of (2.3) satisfy $\|x(t)\| \leq N e^{-\nu t}\left\|x^{0}\right\|$ for all $x^{0} \in X$ and all $t \geq 0$.

We see that the above result is of the same form as Theorem 3.1. In particular, no assumptions are made on the specific structure of the stabilizing continuous-time feedback $F$. It is therefore clear that Remark 3.4 also applies to Theorem 4.8 .

The following lemma is the key tool for the proof of Theorem 4.8

Lemma 4.9. For $\eta>0$, define $W(\eta):=\left\{z=e^{s}\left|s \in \mathbb{C}_{0},\right| s \mid<\eta\right\}$. Under the assumptions of Theorem 4.8, there exist $\eta>0$ and $\theta>0$ such that

$$
\left(I-\mathbf{H}_{\tau}\right)^{-1} \in H^{\infty}(W(\eta), \mathcal{B}(U)) \quad \forall \tau \in(0, \theta),
$$

where $\mathbf{H}_{\tau}$ is given by (4.21).

We defer the long and technical proof of Lemma 4.9 to the end of this section.

Proof of Theorem 4.8. By Lemma 2.3 and Lemma 4.7 it is sufficient to show that there exists $\tau^{*}>0$ such that

$$
\left(I-\mathbf{H}_{\tau}\right)^{-1} \in H^{\infty}(\mathbb{E}, \mathcal{B}(U)), \quad \forall \tau \in\left(0, \tau^{*}\right) .
$$

To this end, let $M \geq 1$ and $\mu \in \mathbb{R}$ be such that

$$
\|T(t)\| \leq M e^{\mu t}, \quad \forall t \in \mathbb{R}_{+} .
$$

We split $\mathbb{E}$ into three disjoint parts:

$$
\mathbb{E}=E_{1}(\delta) \cup E_{2}(\delta) \cup E_{3},
$$


where, for $\delta \in(0,1)$,

$$
\begin{aligned}
& E_{1}(\delta):=\{z \in \mathbb{C}|1<| z|<4 M,| z-1 \mid \leq \delta\}, \\
& E_{2}(\delta):=\{z \in \mathbb{C}|1<| z|<4 M,| z-1 \mid>\delta\},
\end{aligned}
$$

and $E_{3}:=\{z \in \mathbb{C}|| z \mid \geq 4 M\}$. We proceed in several steps.

Step 1: We claim that there exist $\delta>0$ and $\tau_{1}>0$ such that

$$
\left(I-\mathbf{H}_{\tau}\right)^{-1} \in H^{\infty}\left(E_{1}(\delta), \mathcal{B}(U)\right), \quad \forall \tau \in\left(0, \tau_{1}\right) .
$$

By Lemma 4.9 there exist $\eta>0$ and $\theta>0$ such that (4.26) holds. Choosing $\delta \in(0,1)$ such that $E_{1}(\delta) \subset W(\eta)$, we see that (4.30) follows with $\tau_{1}=\theta$.

Step 2: Let $\delta \in(0,1)$ be as in Step 1. We claim that there exists $\tau_{2}>0$ such that

$$
\left(I-\mathbf{H}_{\tau}\right)^{-1} \in H^{\infty}\left(E_{2}(\delta), \mathcal{B}(U)\right), \quad \forall \tau \in\left(0, \tau_{2}\right) .
$$

To this end, first note that by (4.23)

$$
\mathbf{H}_{\tau}^{1}(z)=F_{1}\left((z-1) I-\left(T_{1}(\tau)-I\right)\right)^{-1} \int_{0}^{\tau} T_{1}(s) B_{1} d s .
$$

Since $T_{1}(t)$ is a semigroup on the finite-dimensional space $X_{1}$, we have

$$
\lim _{\tau \rightarrow 0}\left\|T_{1}(\tau)-I\right\|=0 \text {. }
$$

Moreover,

$$
\lim _{\tau \rightarrow 0}\left\|\int_{0}^{\tau} T_{1}(s) B_{1} d s\right\|=0 .
$$

Since $|z-1|>\delta>0$ for all $z \in E_{2}(\delta)$, a routine argument involving the application of the Neumann series shows that there there exists $\theta^{*}>0$ such that

$$
\left.\| \mathbf{H}_{\tau}^{1}(z)\right) \| \leq 1 / 4, \quad \forall z \in E_{2}(\delta), \forall \tau \in\left(0, \theta^{*}\right) .
$$

Now we analyze $\mathbf{H}_{\tau}^{2}(z)$ on $E_{2}^{c l}(\delta)$, the closure of $E_{2}(\delta)$. Using analyticity and boundedness of the semigroup $T_{2}(t)$ (guaranteed by (D4)), an application of Theorem 5.6 (b) on p. 66 in Pazy [14] shows that for each $\lambda \in E_{2}^{c l}(\delta)$ there exist $\theta_{\lambda}>0$ and $k_{\lambda}>0$ such that

$$
\left\|\left(\lambda I-T_{2}(\tau)\right)^{-1}\right\| \leq k_{\lambda}, \quad \forall \tau \in\left(0, \theta_{\lambda}\right) .
$$

For $z, \lambda \in E_{2}^{c l}(\delta)$ we have

$$
\left\|\left(z I-T_{2}(\tau)\right)^{-1}\right\| \leq\left\|\left(T_{2}(\tau)-\lambda I\right)^{-1}\right\|\left\|\left(I-(z-\lambda)\left(T_{2}(\tau)-\lambda I\right)^{-1}\right)^{-1}\right\| .
$$

Combining this with (4.33), an application of the Neumann series yields that for each $\lambda \in E_{2}^{c l}(\delta)$,

$$
\left\|\left(z I-T_{2}(\tau)\right)^{-1}\right\| \leq 2 k_{\lambda}, \quad \forall z \in \mathbb{B}\left(\lambda, 1 /\left(2 k_{\lambda}\right)\right), \forall \tau \in\left(0, \theta_{\lambda}\right) .
$$

Since $E_{2}^{c l}(\delta)$ is compact, we may conclude from a standard compactness argument that there exist $k>0$ and $\tilde{\theta}>0$ such that

$$
\left\|\left(z I-T_{2}(\tau)\right)^{-1}\right\| \leq k, \quad \forall z \in E_{2}^{c l}(\delta), \tau \in(0, \tilde{\theta}) .
$$

By 4.23),

$$
\mathbf{H}_{\tau}^{2}(z)=F_{2}\left(T_{2}(\tau)-I\right)\left(z I-T_{2}(\tau)\right)^{-1} A_{2}^{-1} B_{2},
$$

and since $F_{2}$ is compact, it follows from Lemma 2.1 and (4.34) that there exists $\hat{\theta} \in(0, \tilde{\theta})$ such that

$$
\left\|\mathbf{H}_{\tau}^{2}(z)\right\| \leq 1 / 4, \quad \forall z \in E_{2}(\delta), \tau \in(0, \hat{\theta}) .
$$


Combining this with (4.32) and (4.22) shows that

$$
\left\|\mathbf{H}_{\tau}(z)\right\| \leq 1 / 2, \quad \forall z \in E_{2}(\delta), \tau \in\left(0, \tau_{2}\right),
$$

where $\tau_{2}:=\min \left(\theta^{*}, \hat{\theta}\right)$. Yet another application of the Neumann series yields (4.31).

Step 3: We claim that there exists $\tau_{3}>0$ such that

$$
\left(I-\mathbf{H}_{\tau}\right)^{-1} \in H^{\infty}\left(E_{3}, \mathcal{B}(U)\right), \quad \forall \tau \in\left(0, \tau_{3}\right) .
$$

By (2.10) and (4.28), together with Lemma 2.2, there exists $\tau_{3}>0$ such that

$$
\left\|\Delta_{\tau}\right\| \leq 2 M, \quad \forall \tau \in\left(0, \tau_{3}\right) .
$$

Defining the open set

$$
\tilde{E}_{3}:=\{z \in \mathbb{C}|| z \mid>3 M\} \supset E_{3},
$$

a routine argument involving the Neumann series then shows that

$$
\left\|\left(z I-\Delta_{\tau}\right)^{-1}\right\|=\left|z^{-1}\right|\left\|\left(I-z^{-1} \Delta_{\tau}\right)^{-1}\right\| \leq 1 / M, \quad \forall z \in \tilde{E}_{3}, \forall \tau \in\left(0, \tau_{3}\right) .
$$

Now

$$
\left(I-\mathbf{H}_{\tau}(z)\right)^{-1}=I+F\left(z I-\Delta_{\tau}\right)^{-1} S_{\tau},
$$

and so (4.35) follows from (4.36), (4.37) and Lemma 2.2.

Step 4: Finally, setting $\tau^{*}=\min \left(\tau_{1}, \tau_{2}, \tau_{3}\right)$, a combination of (4.29)-(4.31) and (4.35) yields 4.27).

It remains to prove the crucial Lemma 4.9.

Proof of Lemma 4.9. Recall the definition of $\mathbf{G}$ in (4.2). Appealing to (D1)-(D4), (4.13) and (4.14), we may write

$$
\mathbf{G}=\mathbf{G}_{1}+\mathbf{G}_{2}, \text { where } \mathbf{G}_{j}(s)=F_{j}\left(s I-A_{j}\right)^{-1} B_{j}, j=1,2 .
$$

It is convenient to set

$$
\mathbb{C}_{0}^{*}:=\mathbb{C}_{0} \backslash \sigma(A)=\mathbb{C}_{0} \backslash \sigma\left(A_{1}\right) .
$$

For $\eta>0$ and $\theta>0$, we define

$$
\begin{aligned}
V(\theta, \eta) & :=\left\{(\tau, s) \in(0, \infty) \times \mathbb{C}_{0}|\tau \in(0, \theta), \tau| s \mid<\eta\right\}, \\
V^{*}(\theta, \eta) & :=\left\{(\tau, s) \in(0, \infty) \times \mathbb{C}_{0}^{*}|\tau \in(0, \theta), \tau| s \mid<\eta\right\} .
\end{aligned}
$$

The idea of the proof is to compare $\mathbf{H}_{\tau}\left(e^{\tau s}\right)$ to $\mathbf{G}(s)$ for all $(\tau, s) \in V^{*}(\theta, \eta)$, where $\eta>0$ and $\theta>0$ are sufficiently small. We proceed in several steps.

Step 1: In this step we analyze the term $\mathbf{H}_{\tau}^{1}\left(e^{\tau s}\right)-\mathbf{G}_{1}(s)$. We claim that

$$
\mathbf{G}_{1}(s)-\mathbf{H}_{\tau}^{1}\left(e^{\tau s}\right)=F_{1}\left(s I-A_{1}\right)^{-1} Q(\tau, s) B_{1}, \quad \forall(\tau, s) \in(0, \infty) \times \mathbb{C}_{0}^{*},
$$

where $Q$ is a matrix-valued function defined on $(0, \infty) \times \mathbb{C}_{0}^{*}$ such that

$$
\lim _{(\theta, \eta) \rightarrow 0}\left(\sup \left\{\|Q(\tau, s)\| \mid(\tau, s) \in V^{*}(\theta, \eta)\right\}\right)=0 .
$$

Using the finite-dimensionality of $X_{1}$, we obtain using Taylor expansions

$$
\int_{0}^{\tau} T_{1}(t) B_{1} d t=\tau\left(I+Q_{1}(\tau)\right) B_{1}, \quad I-e^{-\tau s} T_{1}(\tau)=\tau\left(I+Q_{2}(\tau, s)\right)\left(s I-A_{1}\right),
$$

where

$$
Q_{1}(\tau):=\sum_{k=2}^{\infty} \frac{\tau^{k-1}}{k !} A_{1}^{k-1}, \quad Q_{2}(\tau, s):=\sum_{k=2}^{\infty} \frac{\tau^{k-1}}{k !}\left(A_{1}-s I\right)^{k-1}
$$


Note that $Q_{1}$ and $Q_{2}$ satisfy

$$
\lim _{\tau \rightarrow 0} Q_{1}(\tau)=0, \quad \lim _{(\tau, \tau s) \rightarrow 0} Q_{2}(\tau, s)=0 .
$$

The first limit follows immediately from the definition of $Q_{1}$, whilst the second limit follows from the inequality

$$
\left\|Q_{2}(\tau, s)\right\| \leq e^{\tau\left(|s|+\left\|A_{1}\right\|\right)}-1,
$$

which is the result of a routine estimate. Using (4.41), a straightforward calculation shows that (4.39) holds with

$$
Q(\tau, s):=\tau\left[\left(1-e^{-\tau s}\right) I-e^{-\tau s} Q_{1}(\tau)+Q_{2}(\tau, s)\right]\left(s I-A_{1}\right)\left(I-e^{-\tau s} T_{1}(\tau)\right)^{-1} .
$$

Note that, by the spectral mapping theorem, $I-e^{-\tau s} T_{1}(\tau)$ is invertible for all $(\tau, s) \in(0, \infty) \times \mathbb{C}_{0}^{*}$, and so, $Q(\tau, s)$ is well-defined for all $(\tau, s) \in(0, \infty) \times \mathbb{C}_{0}^{*}$. From (4.41) we obtain

$$
Q(\tau, s)=\left[\left(1-e^{-\tau s}\right) I-e^{-\tau s} Q_{1}(\tau)+Q_{2}(\tau, s)\right]\left(I+Q_{2}(\tau, s)\right)^{-1},
$$

and it follows from (4.42) that $Q$ satisfies (4.40).

Step 2: We show that

$$
\lim _{(\theta, \eta) \rightarrow 0}\left(\sup \left\{\left\|\mathbf{H}_{\tau}^{2}\left(e^{\tau s}\right)-\mathbf{G}_{2}(s)\right\| \mid(\tau, s) \in V(\theta, \eta)\right\}\right)=0 .
$$

In order to estimate the difference $\mathbf{H}_{\tau}^{2}\left(e^{\tau s}\right)-\mathbf{G}_{2}(s)$, note that

$$
\begin{aligned}
\mathbf{H}_{\tau}^{2}\left(e^{\tau s}\right)-\mathbf{G}_{2}(s)= & F_{2}\left[\left(e^{\tau s} I-T_{2}(\tau)\right)^{-1}\left(T_{2}(\tau)-I\right) A_{2}^{-1}-\left(s I-A_{2}\right)^{-1}\right] B_{2} \\
=F_{2}[ & \left.\left(T_{2}(\tau)-I\right) A_{2}^{-1}\left(s I-A_{2}\right)-\left(e^{\tau s} I-T_{2}(\tau)\right)\right] \\
& \times\left(e^{\tau s} I-T_{2}(\tau)\right)^{-1}\left(s I-A_{2}\right)^{-1} B_{2} .
\end{aligned}
$$

Setting $R_{2}(s):=\left(s I-A_{2}\right)^{-1}$, routine algebraic manipulations show that

$$
\mathbf{H}_{\tau}^{2}\left(e^{\tau s}\right)-\mathbf{G}_{2}(s)=P_{1}(\tau, s) P_{2}(\tau, s),
$$

where

$$
\begin{aligned}
& P_{1}(\tau, s):=F_{2}\left(e^{-\tau s} \frac{T_{2}(\tau)-I}{\tau} A_{2}^{-1}-\frac{1-e^{-\tau s}}{\tau s} I\right), \\
& P_{2}(\tau, s):=\left(\frac{I-e^{-\tau s} T_{2}(\tau)}{\tau s}\right)^{-1} R_{2}(s) B_{2} .
\end{aligned}
$$

We first analyze $P_{1}(\tau, s)$. Since the term

$$
e^{-\tau s} \frac{T_{2}(\tau)-I}{\tau} A_{2}^{-1}-\frac{1-e^{-\tau s}}{\tau s} I
$$

converges strongly to 0 as $(\tau, \tau s) \rightarrow 0$, the compactness of $F_{2}$ together with Lemma 2.1 shows that

$$
\lim _{(\tau, \tau s) \rightarrow 0}\left\|P_{1}(\tau, s)\right\|=0 .
$$

Estimating $P_{2}(\tau, s)$ is considerably more difficult. In view of (4.45) and 4.46), to establish (4.44), it is sufficient to prove the following claim.

Claim: For any $p \in(0, \pi)$ there exist $\delta>0$ and $k>0$ such that

$$
\left\|P_{2}(\tau, s)\right\| \leq k<\infty, \quad \forall(\tau, s) \in V(\delta, p) .
$$


Proof. Since the semigroup $T_{2}(t)$ is exponentially stable, for fixed $s \in \mathbb{C}_{0}$ and $\tau>0$ the spectral radius of $e^{-\tau s} T_{2}(\tau)$ is smaller than 1 , and the Neumann series gives

$$
\left(I-e^{-\tau s} T_{2}(\tau)\right)^{-1}=\sum_{k=0}^{\infty} e^{-k \tau s} T_{2}(k \tau) .
$$

Since $T_{2}(t)$ is an exponentially stable analytic semigroup, there exist $\beta \in(0, \pi / 2)$, $\omega>0$ and $M>0$ such that

$$
\varrho\left(A_{2}\right) \supset \Xi:=\{\xi \in \mathbb{C}|| \arg (\xi+\omega) \mid<\pi / 2+\beta\}
$$

and

$$
\left\|R_{2}(\xi)\right\| \leq M /|\xi|, \quad \forall \xi \in \Xi \backslash\{0\} .
$$

If we note that $R_{2}(\xi) B_{2}=\left(\xi R_{2}(\xi)-I\right) A_{2}^{-1} B_{2}$, (4.49) shows that for any $\varepsilon>0$ there exists $N>0$ such that

$$
\left\|R_{2}(\xi) B_{2}\right\|_{\mathcal{B}(U, X)} \leq N, \quad \forall \xi \in \Xi .
$$

Let $\psi \in(\pi / 2, \pi / 2+\beta)$ and $r_{0} \in(0, \omega)$. Define the contour

$$
\Gamma=\left\{r e^{-i \psi} \mid r>r_{0}\right\} \cup\left\{r_{0} e^{i \phi} \mid \phi \in[\psi, 2 \pi-\psi]\right\} \cup\left\{r e^{i \psi} \mid r>r_{0}\right\},
$$

oriented bottom to top. Note that by construction $\Gamma \subset \Xi$ and $\sigma\left(A_{2}\right)$ is to the left of $\Gamma$; see Figure 4.1

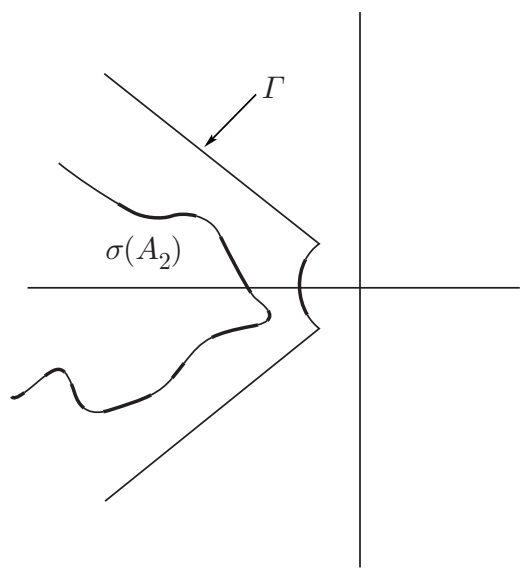

Figure 4.1. The contour $\Gamma$ with $\sigma\left(A_{2}\right)$ to the left of $\Gamma$

Invoking (4.48) and using a standard relationship between $T_{2}(t)$ and $R_{2}(s)$ (see [14, Theorem 7.7, p. 30), we have

$$
\left(I-e^{-\tau s} T_{2}(\tau)\right)^{-1}=I+\frac{1}{2 \pi i} \sum_{k=1}^{\infty} \int_{\Gamma} e^{-k \tau s} e^{k \tau \lambda} R_{2}(\lambda) d \lambda .
$$

By (4.49),

$$
\left\|e^{-k \tau s} e^{k \tau \lambda} R_{2}(\lambda)\right\| \leq \frac{M}{|\lambda|}\left|e^{k \tau(\lambda-s)}\right|, \quad \forall \lambda \in \Gamma, \forall s \in \mathbb{C}_{0}, \forall \tau>0 .
$$


Hence we can use Lebesgue's dominated convergence theorem to interchange the order of summation and integration in (4.51). Evaluating the resulting infinite sum, we obtain

$$
P_{2}(\tau, s)=\tau s R_{2}(s) B_{2}+\frac{1}{2 \pi i} \int_{\Gamma} \frac{\tau s e^{\tau(\lambda-s)}}{1-e^{\tau(\lambda-s)}} R_{2}(\lambda) R_{2}(s) B_{2} d \lambda .
$$

Setting

$$
f(\tau, \lambda, s):=\frac{\tau s e^{\tau(\lambda-s)}}{1-e^{\tau(\lambda-s)}}+\frac{s}{\lambda-s}=\frac{\tau s}{e^{\tau(s-\lambda)}-1}-\frac{s}{s-\lambda},
$$

and letting $p \in(0, \pi)$ be fixed, but arbitrary, an elementary analysis (see the Appendix) shows that

$$
\sup \left\{\left|\frac{f(\tau, \lambda, s)}{\tau(s-\lambda)}\right| \mid(\tau, \lambda, s) \in(0, \infty) \times \Gamma \times \mathbb{C}_{0} \quad \text { s.t. } \tau|\lambda| \leq p, \tau|s| \leq p\right\}<\infty .
$$

Define $\delta:=p / r_{0}$ and for $\tau \in(0, \delta)$ introduce

$$
\Gamma_{\tau}:=\{\lambda \in \Gamma|\tau| \lambda \mid \leq p\} .
$$

Clearly, $\Gamma_{\tau} \neq \emptyset$ for all $\tau \in(0, \delta)$. We decompose the integral on the right-hand side of (4.52) as follows:

$$
I(\tau, s):=\int_{\Gamma} \frac{\tau s e^{\tau(\lambda-s)}}{1-e^{\tau(\lambda-s)}} R_{2}(\lambda) R_{2}(s) B_{2} d \lambda=I_{1}(\tau, s)+I_{2}(\tau, s) R_{2}(s) B_{2},
$$

where

$$
I_{1}(\tau, s):=\int_{\Gamma_{\tau}} \frac{\tau s e^{\tau(\lambda-s)}}{1-e^{\tau(\lambda-s)}} R_{2}(\lambda) R_{2}(s) B_{2} d \lambda, \quad I_{2}(\tau, s):=\int_{\Gamma \backslash \Gamma_{\tau}} \frac{\tau s e^{\tau(\lambda-s)}}{1-e^{\tau(\lambda-s)}} R_{2}(\lambda) d \lambda .
$$

We first analyze $I_{1}(\tau, s)$. Using the resolvent identity

$$
(s-\lambda) R_{2}(\lambda) R_{2}(s)=R_{2}(\lambda)-R_{2}(s),
$$

and invoking (4.53), we obtain

$$
I_{1}(\tau, s)=\int_{\Gamma_{\tau}} f(\tau, \lambda, s) \frac{R_{2}(\lambda)-R_{2}(s)}{s-\lambda} B_{2} d \lambda+\int_{\Gamma_{\tau}} \frac{s}{s-\lambda} R_{2}(\lambda) R_{2}(s) B_{2} d \lambda
$$

Setting

$$
I_{11}(\tau, s):=\int_{\Gamma_{\tau}} f(\tau, \lambda, s) \frac{R_{2}(\lambda)-R_{2}(s)}{s-\lambda} B_{2} d \lambda, \quad I_{12}(\tau, s):=\int_{\Gamma_{\tau}} \frac{s}{s-\lambda} R_{2}(\lambda) d \lambda,
$$

we have

$$
I_{1}(\tau, s)=I_{11}(\tau, s)+I_{12} R_{2}(s) B_{2} .
$$

Invoking (4.50) and (4.54) shows that there exists $k_{1}>0$ such that for all $(\tau, s) \in$ $V(\delta, p)$,

$$
\begin{aligned}
\left\|I_{11}(\tau, s)\right\| & \leq \int_{\Gamma_{\tau}} \frac{|f(\tau, \lambda, s)|}{|\lambda-s|}\left(\left\|R_{2}(\lambda) B_{2}\right\|+\left\|R_{2}(s) B_{2}\right\|\right) d|\lambda| \\
& \leq k_{1} \tau \text { length }\left(\Gamma_{\tau}\right) .
\end{aligned}
$$

Combining this with

$$
\text { length }\left(\Gamma_{\tau}\right) \leq 2(\pi+1) p / \tau
$$

and setting $k_{2}:=2 k_{1}(\pi+1) p$ yields

$$
\left\|I_{11}(\tau, s)\right\| \leq k_{2}, \quad \forall(\tau, s) \in V(\delta, p) .
$$


To estimate $I_{12}(\tau, s)$, we define

$$
\kappa(\tau):=2 p / \tau,
$$

and for each $\tau \in(0, \delta)$ we embed $\Gamma_{\tau}$ into a closed contour

$$
\begin{aligned}
C_{\tau}:= & \left\{r e^{-i \psi} \mid r \in\left[r_{0}, \kappa(\tau)\right]\right\} \cup\left\{r_{0} e^{i \phi} \mid \phi \in[\psi, 2 \pi-\psi]\right\} \\
& \cup\left\{r e^{i \psi} \mid r \in\left[r_{0}, \kappa(\tau)\right]\right\} \cup\left\{\kappa(\tau) e^{i \phi} \mid \phi \in[-\psi, \psi]\right\},
\end{aligned}
$$

oriented clockwise (see Figure 4.2). Then

$$
\begin{aligned}
I_{12}(\tau, s) & =\int_{C_{\tau}} \frac{s}{(s-\lambda)} R_{2}(\lambda) d \lambda-\int_{p / \tau}^{\kappa(\tau)} \frac{s}{s-r e^{i \psi}} R_{2}\left(r e^{i \psi}\right) e^{i \psi} d r \\
(4.59) & +\int_{p / \tau}^{\kappa(\tau)} \frac{s}{s-r e^{-i \psi}} R_{2}\left(r e^{-i \psi}\right) e^{-i \psi} d r+\int_{-\psi}^{\psi} \frac{i s \kappa(\tau) e^{i \phi}}{s-\kappa(\tau) e^{i \phi}} R_{2}\left(\kappa(\tau) e^{i \phi}\right) d \phi .
\end{aligned}
$$

By construction, for a given $\tau \in(0, \delta)$, any $s \in \mathbb{C}_{0} \cap \mathbb{B}(0, p / \tau)$ is inside the contour $C_{\tau}$ and so Cauchy's integral formula combined with (4.49) shows that there exists $k_{3}>0$ such that

$$
\left\|\int_{C_{\tau}} \frac{s}{(s-\lambda)} R_{2}(\lambda) d \lambda\right\|=2 \pi\left\|s R_{2}(s)\right\| \leq k_{3}, \quad \forall(\tau, s) \in V(\delta, p) .
$$

Moreover, since $\left|s-r e^{i \psi}\right|>r|\cos \psi|$ for $s \in \mathbb{C}_{0}$, it follows from (4.49) that there exists $k_{4}>0$ such that

$$
\begin{aligned}
\left\|\int_{p / \tau}^{\kappa(\tau)} \frac{s}{s-r e^{i \psi}} R_{2}\left(r e^{i \psi}\right) e^{i \psi} d r\right\| & \leq \frac{k_{4}|s|}{|\cos \psi|} \int_{p / \tau}^{\infty} \frac{d r}{r^{2}} \\
& =\frac{k_{4}}{p|\cos \psi|} \tau|s|, \quad \forall(\tau, s) \in V(\delta, p) .
\end{aligned}
$$

As an immediate consequence we see that

$$
\left\|\int_{p / \tau}^{\kappa(\tau)} \frac{s}{s-r e^{i \psi}} R_{2}\left(r e^{i \psi}\right) e^{i \psi} d r\right\| \leq k_{5}, \quad \forall(\tau, s) \in V(\delta, p),
$$

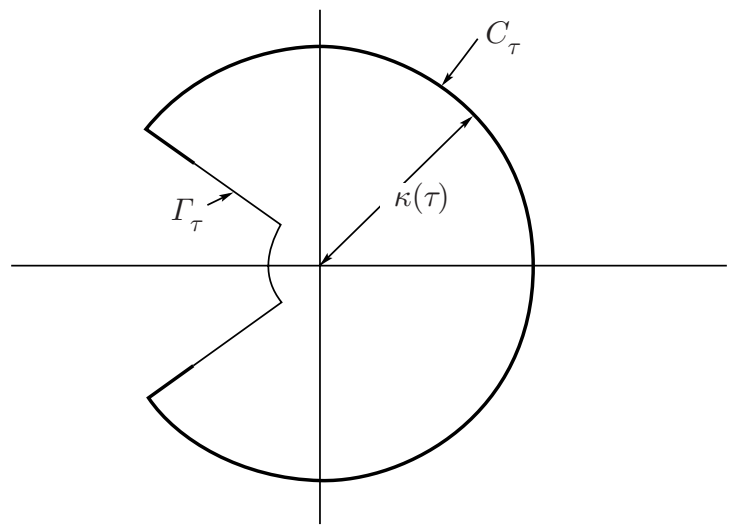

Figure 4.2. The contour $\Gamma_{\tau}$ embedded in a closed contour $C_{\tau}$ 
for some suitable $k_{5}>0$. Similarly, there exists $k_{6}>0$ such that

$$
\left\|\int_{p / \tau}^{\kappa(\tau)} \frac{s}{s-r e^{-i \psi}} R_{2}\left(r e^{-i \psi}\right) e^{-i \psi} d r\right\| \leq k_{6}, \quad \forall(\tau, s) \in V(\delta, p) .
$$

Trivially, since $\kappa(\tau)=2 p / \tau$,

$$
\left|s-\kappa(\tau) e^{i \phi}\right| \geq p / \tau, \quad \forall(\tau, s) \in V(\delta, p), \forall \phi \in[-\psi, \psi],
$$

and hence, using (4.49), we see that there exists $k_{7}>0$ such that

$$
\begin{aligned}
\left\|\int_{-\psi}^{\psi} \frac{i s \kappa(\tau) e^{i \phi}}{s-\kappa(\tau) e^{i \phi}} R_{2}\left(\kappa(\tau) e^{i \phi}\right) d \phi\right\| & \leq \frac{k_{7} \tau|s|}{p} \int_{-\psi}^{\psi} d \phi=\frac{2 k_{7} \psi \tau|s|}{p} \\
& \leq 2 k_{7} \psi, \quad \forall(\tau, s) \in V(\delta, p) .
\end{aligned}
$$

Combining (4.59)-(4.63) shows that there exists $k_{8}>0$ such that

$$
\left.\| I_{12}(\tau, s)\right) \| \leq k_{8}, \quad \forall(\tau, s) \in V(\delta, p) .
$$

By (4.50) we may conclude that $I_{12}(\tau, s) R_{2}(s) B_{2}$ is uniformly bounded on $V(\delta, p)$, and therefore, by (4.57) and (4.58), we obtain that there exists $k_{9}>0$ such that

$$
\left.\| I_{1}(\tau, s)\right) \| \leq k_{9}, \quad \forall(\tau, s) \in V(\delta, p) .
$$

To estimate $I_{2}(\tau, s)$, first note that there exists $k_{10}>0$ such that

$$
\left|1-e^{\tau(\lambda-s)}\right| \geq k_{10}>0, \quad \forall(\tau, \lambda, s) \in(0, \infty) \times \Gamma \times \mathbb{C}_{0} \text { s.t. } \lambda \in \Gamma \backslash \Gamma_{\tau} .
$$

Combining this with an application of (4.49), we obtain

$$
\left\|I_{2}(\tau, s)\right\| \leq \frac{2 k_{11} p}{k_{10}} \int_{p / \tau}^{\infty} \frac{e^{\tau r \cos \psi}}{r} d r, \quad \forall(\tau, s) \in V(\delta, p),
$$

for some suitable constant $k_{11}>0$. Since $\cos \psi<0$,

$$
\int_{p / \tau}^{\infty} \frac{e^{\tau r \cos \psi}}{r} d r \leq \frac{\tau}{p \tau|\cos \psi|} e^{p \cos \psi} \leq \frac{e^{p \cos \psi}}{p|\cos \psi|}<\infty .
$$

Together with (4.66) this shows that there exists $k_{12}>0$ such that

$$
\left\|I_{2}(\tau, s)\right\| \leq k_{12}, \quad \forall(\tau, s) \in V(\delta, p) .
$$

Combining (4.52), (4.55), (4.65) and (4.67) and invoking (4.50) shows that (4.47) holds, completing the proof of the claim.

Step 3: By assumption, the analytic semigroup generated by $A_{B F}$ is exponentially stable. Therefore, by Proposition 4.5 .

$$
(I-\mathbf{G})^{-1} \in H^{\infty}\left(\mathbb{C}_{0}, \mathcal{B}(U)\right),
$$

which, in particular, implies that $I-\mathbf{G}(s)$ is invertible for all $s \in \mathbb{C}_{0}^{*}$. Setting

$$
D(\tau, s):=\mathbf{G}_{2}(s)-\mathbf{H}_{\tau}^{2}\left(e^{s \tau}\right), \quad \forall(\tau, s) \in(0, \infty) \times \mathbb{C}_{0},
$$

and

$$
\tilde{Q}(\tau, s):=\left(\begin{array}{cc}
Q(\tau, s) & 0 \\
0 & 0
\end{array}\right) \in \mathcal{B}\left(X_{-1}, X\right), \quad \forall(\tau, s) \in(0, \infty) \times \mathbb{C}_{0}^{*},
$$


with $Q$ given by (4.43), we obtain from (4.38) and (4.39) for all $(\tau, s) \in(0, \infty) \times \mathbb{C}_{0}^{*}$ that

$$
\begin{aligned}
\left.I-\mathbf{H}_{\tau}\left(e^{\tau s}\right)\right)= & I-\mathbf{G}_{1}(s)-\mathbf{G}_{2}(s)+F_{1}\left(s I-A_{1}\right)^{-1} Q(\tau, s) B_{1}+D(\tau, s) \\
= & (I-\mathbf{G}(s))\left[I+(I-\mathbf{G}(s))^{-1} F(s I-A)^{-1} \tilde{Q}(s, \tau) B\right. \\
& \left.+(I-\mathbf{G}(s))^{-1} D(\tau, s)\right] .
\end{aligned}
$$

A straightforward calculation combined with an application of statements (2) and (3) of Lemma 4.1 shows that

$$
\begin{aligned}
(I-\mathbf{G}(s))^{-1} F(s I-A)^{-1} x & =F(s I-A-B F)^{-1} x \\
& =F\left(s I-A_{B F}\right)^{-1} x, \quad \forall s \in \mathbb{C}_{0}^{*}, \forall x \in X .
\end{aligned}
$$

Moreover, by 4.70),

$$
(\tilde{Q}(\tau, s) B) U \subset X, \quad \forall(\tau, s) \in(0, \infty) \times \mathbb{C}_{0}^{*} .
$$

Combining (4.71)-(4.73) shows that for all $(\tau, s) \in(0, \infty) \times \mathbb{C}_{0}^{*}$,

$$
I-\mathbf{H}_{\tau}\left(e^{\tau s}\right)=(I-\mathbf{G}(s))\left[I+F\left(s I-A_{B F}\right)^{-1} \tilde{Q}(\tau, s) B+(I-\mathbf{G}(s))^{-1} D(\tau, s)\right] .
$$

By assumption, the analytic semigroup generated by $A_{B F}$ is exponentially stable. Consequently, the function $s \mapsto\left(s I-A_{B F}\right)^{-1}$ is in $H^{\infty}\left(\mathbb{C}_{0}, \mathcal{B}(X)\right)$, and thus we may conclude from (4.40) and (4.70) that

$$
\lim _{(\theta, \eta) \rightarrow 0}\left(\sup \left\{\left\|F\left(s I-A_{B F}\right)^{-1} \tilde{Q}(\tau, s) B\right\| \mid(\tau, s) \in V^{*}(\theta, \eta)\right\}\right)=0 .
$$

From (4.44) and (4.69) combined with (4.68), we obtain

$$
\left.\lim _{(\theta, \eta) \rightarrow 0}\left(\sup \left\{\|(I-\mathbf{G}(s))^{-1} D(\tau, s)\right] \| \mid(\tau, s) \in V(\theta, \eta)\right\}\right)=0 .
$$

Finally, combining (4.74)-(4.76) shows that that there exist $\theta>0$ and $\eta>0$ such that 4.26) holds.

\section{Examples}

In this section we give examples which illustrate Theorem 4.8 We consider a general parabolic partial differential equation with Dirichlet control. For two different types of stabilizing feedbacks $u=F x$ found in the literature on control of partial differential equations, we show that the associated sampled-data feedback (1.2) stabilizes the system for small enough $\tau>0$.

Let $\Omega$ be a convex bounded open set in $\mathbb{R}^{n}, n \geq 2$, with $C^{\infty}$ boundary $\Gamma$. Let

$$
L(\xi, \partial)=\sum_{|\alpha| \leq 2} a_{\alpha}(\xi) \partial^{\alpha}
$$

with smooth real coefficients $a_{\alpha}$, where $\partial$ is the spatial derivative operator. We consider the following parabolic system:

$$
\frac{\partial x}{\partial t}(t, \xi)=-L(\xi, \partial) x(t, \xi) \text { in }(0, \infty) \times \Omega, x(t, \zeta)=u(t, \zeta) \text { in }(0, \infty) \times \Gamma .
$$

An example of such a system is a heat equation in a disk.

Let $X=L^{2}(\Omega), U=L^{2}(\Gamma)$, and let $A$ be the operator $-L(\xi, \partial)$ with domain $\mathcal{D}(A)=\left\{x \in H^{2}(\Omega)|x|_{\Gamma}=0\right\}$. It is well known that $A$ is the generator of an analytic semigroup; see for instance [4]. Define the Dirichlet map $D$ by

$$
x=D y \text { if } L(\xi, \partial) x=0 \text { in } \Omega \text { and } x=y \text { on } \Gamma .
$$


Feedback stabilization of (5.1) is studied in Lasiecka and Triggiani [10, [11, [12]. In 11] it is shown that (5.1) can be written in the form $\dot{x}=A x+B u$ with $B=-A D$. From standard elliptic theory, $D \in \mathcal{B}\left(L^{2}(\Gamma), H^{1 / 2}(\Omega)\right.$ ) (see for instance [13]); so we see that $B \in \mathcal{B}\left(U, X_{-1}\right)$.

We now describe two examples of compact feedbacks that exponentially stabilize (5.1).

Example 1. We first consider the rank $m$ feedback from [10] of the form

$$
F x(t, \cdot)=\sum_{j=1}^{m}\left\langle x(t, \cdot), w_{j}(\cdot)\right\rangle_{L^{2}(\Omega)} g_{j}(\cdot),
$$

where $w_{j} \in L^{2}(\Omega)$ and $g_{j} \in L^{2}(\Gamma)$. Theorem 1.2 from [10] gives conditions on $w_{j}$ and $g_{j}$ that guarantee that $A+B F$ generates an exponentially stable semigroup. Since $F$ is obviously compact, we can apply Theorem 4.8 to conclude that (1.2) exponentially stabilizes (5.1) for small enough $\tau>0$.

Example 2. Here the stabilizing feedback arises from the solution of a linear quadratic optimal control problem. Consider the cost functional

$$
J(u, x)=\int_{0}^{\infty}\left(\|u(t)\|_{L^{2}(\Gamma)}^{2}+\|x(t)\|_{L^{2}(\Omega)}^{2}\right) d t
$$

where $x$ is the solution of (5.1) corresponding to the control function $u$. It is shown in [11] that the optimal control is given by the feedback

$$
u=\frac{\partial}{\partial \nu_{A^{*}}} P x,
$$

where $\partial / \partial \nu_{A^{*}}$ is the co-normal derivative with respect to $A^{*}$ and $P \in \mathcal{B}\left(L^{2}(\Omega)\right)$ is a nonnegative selfadjoint operator which is the unique solution of the algebraic Riccati equation

$$
\langle x, y\rangle_{L^{2}(\Omega)}+\langle P x, A y\rangle_{L^{2}(\Omega)}+\langle A x, P y\rangle_{L^{2}(\Omega)}=\left\langle\frac{\partial}{\partial \nu_{A^{*}}} P x, \frac{\partial}{\partial \nu_{A^{*}}} P y\right\rangle_{L^{2}(\Gamma)} .
$$

It is shown in Theorem 2.8 in [11] that the control (5.2) can be written as $u=F x$, where $F \in \mathcal{B}(X, U)$. Furthermore, it is shown in the proof of Theorem 2.10 in [11] that $B F$ can be written in the form $A K$, where $K \in \mathcal{B}(X)$ is compact. Hence we can apply Theorem 4.8 to $\dot{x}=A x+A v$ with feedback $v=K x$, to conclude that (1.2) exponentially stabilizes (5.1) for small enough $\tau>0$. In [12], Galerkin approximations of the feedback (5.2) were developed and shown to be exponentially stabilizing for sufficiently small mesh parameters. These Galerkin approximations produce finite-rank feedback, and therefore Theorem 4.8 can again be applied.

\section{Dynamic SAMPled-DATA FEedBaCK}

In this section we apply the results of the previous sections to dynamic sampleddata feedback. For simplicity and the sake of brevity, we restrict ourselves here to systems with bounded control and bounded observation. The system to be controlled, the plant, is formally given by

$$
\begin{aligned}
\dot{x}(t) & =A x(t)+B u(t), t \geq 0 ; x(0)=x^{0} \in X, \\
y(t) & =C x(t),
\end{aligned}
$$


where $A: D(A) \subset X \rightarrow X$ generates a strongly continuous semigroup $T(t), B \in$ $\mathcal{B}(U, X), C \in \mathcal{B}(X, Y)$, and $X, U$ and $Y$ are Hilbert spaces.

Consider a continuous-time controller of the form

$$
\begin{aligned}
\dot{z}(t) & =A_{c} z(t)+B_{c} v(t), t \geq 0 ; z(0)=z^{0} \in X_{c}, \\
w(t) & =C_{c} z(t)+D_{c} v(t),
\end{aligned}
$$

where $A_{c}: D\left(A_{c}\right) \subset X_{c} \rightarrow X_{c}$ generates a strongly continuous semigroup $T_{c}(t)$, $B_{c} \in \mathcal{B}\left(Y, X_{c}\right), C_{c} \in \mathcal{B}\left(X_{c}, U\right), D_{c} \in \mathcal{B}(Y, U)$ and $X_{c}$ is a Hilbert space. Using the output (6.1b) of (6.1) as the input for (6.2) and the output (6.2b) of (6.2) as the input for (6.1), i.e.,

$$
v=y \quad \text { and } \quad u=w,
$$

we obtain the feedback interconnection of (6.1) and (6.2). It is convenient to define

$$
\tilde{A}:=\left(\begin{array}{cc}
A & 0 \\
0 & A_{c}
\end{array}\right), \tilde{B}:=\left(\begin{array}{cc}
B & 0 \\
0 & B_{c}
\end{array}\right), \tilde{C}:=\left(\begin{array}{cc}
C & 0 \\
0 & C_{c}
\end{array}\right), \tilde{K}:=\left(\begin{array}{cc}
D_{c} & I_{U} \\
I_{Y} & 0
\end{array}\right) .
$$

Clearly, $\tilde{A}$ generates the strongly continuous semigroup

$$
\tilde{T}(t):=\left(\begin{array}{cc}
T(t) & 0 \\
0 & T_{c}(t)
\end{array}\right) .
$$

We say that the continuous-time feedback system given by 6.1)-6.3 is exponentially stable if the strongly continuous semigroup generated by

$$
\tilde{A}+\tilde{B} \tilde{K} \tilde{C}=\left(\begin{array}{cc}
A+B D_{c} C & B C_{c} \\
B_{c} C & A_{c}
\end{array}\right)
$$

is exponentially stable.

Let $\tau>0$ and consider the following discretization of (6.2), which is obtained by applying the standard hold and sampling operations to (6.2):

$$
\begin{aligned}
z_{k+1} & =T_{c}(\tau) z_{k}+\int_{0}^{\tau} T_{c}(s) B_{c} v_{k} d s, k \in \mathbb{N}_{0} ; z_{0}=z^{0} \in X_{c}, \\
w_{k} & =C_{c} z_{k}+D_{c} v_{k} .
\end{aligned}
$$

We use the discrete-time system (6.4) to control the continuous-time system (6.1) by sampled-data feedback, i.e., we consider the feedback interconnection of (6.1) and (6.4) given by

$$
v_{k}=y(k \tau) \text { and } u(k \tau+t)=w_{k}, t \in[0, \tau) ; k \in \mathbb{N}_{0} .
$$

Via an application of the variation of parameters formula to (6.1a), this leads to the following sampled-data feedback equations:

$$
\begin{aligned}
x(k \tau+t) & =T(t) x(k \tau)+\int_{0}^{t} T(s) B\left(D_{c} C x(k \tau)+C_{c} z_{k}\right) d s, t \in[0, \tau) ; x(0)=x^{0}, \\
(6.5 \mathrm{~b}) & \\
z_{k+1} & =T_{c}(\tau) z_{k}+\int_{0}^{\tau} T_{c}(s) B_{c} C x(k \tau) d s, \quad k \in \mathbb{N}_{0} ; z_{0}=z^{0} .
\end{aligned}
$$

The sampled-data feedback system (6.5) is called exponentially stable if there exist $N \geq 1$ and $\nu>0$ such that for all initial conditions $\left(x^{0}, z^{0}\right) \in X \times X_{c}$,

$$
\left\|\left(x(k \tau+t), z_{k}\right)\right\| \leq N e^{-\nu(k \tau+t)}\left\|\left(x^{0}, z^{0}\right)\right\|, \quad \forall t \in[0, \tau), \forall k \in \mathbb{N}_{0} .
$$


By a straightforward calculation it follows from (6.4a) that

$$
\left(\begin{array}{c}
x((k+1) \tau) \\
z_{k+1}
\end{array}\right)=\tilde{\Delta}_{\tau}\left(\begin{array}{c}
x(k \tau) \\
z_{k}
\end{array}\right), \quad k \in \mathbb{N}_{0},
$$

where

$$
\tilde{\Delta}_{\tau}: X \times X_{c} \rightarrow X \times X_{c}, \quad \tilde{x} \mapsto \tilde{T}(\tau) \tilde{x}+\int_{0}^{\tau} \tilde{T}(s) \tilde{B} \tilde{K} \tilde{C} \tilde{x} d s .
$$

The proof of the following lemma is a routine exercise and is therefore left to the reader.

Lemma 6.1. For any $\tau>0$, the sampled-data feedback system (6.5) is exponentially stable if and only if $\tilde{\Delta}_{\tau}$ is power stable.

We are now in position to formulate the main result of this section.

Theorem 6.2. Assume that the operator $\tilde{K} \tilde{C}$ is compact. If the continuous-time feedback system (6.1)-(6.3) is exponentially stable (i.e., the strongly continuous semigroup generated by $\tilde{A}+\tilde{B} \tilde{K} \tilde{C}$ is exponentially stable), then there exists $\tau^{*}>0$ such that for every $\tau \in\left(0, \tau^{*}\right)$ the sampled-data feedback system (6.5) is exponentially stable.

Proof. Introducing the operator

$$
\tilde{S}_{\tau}: U \times Y \rightarrow X \times X_{c}, \quad \tilde{u} \mapsto \int_{0}^{\tau} \tilde{T}(s) \tilde{B} \tilde{u} d s,
$$

we may write $\tilde{\Delta}_{\tau}=\tilde{T}(\tau)+\tilde{S}_{\tau} \tilde{K} \tilde{C}$, and we see that $\tilde{\Delta}$ is of the form (2.10). By the compactness of $\tilde{K} \tilde{C}$ and the exponential stability of the semigroup generated by $\tilde{A}+\tilde{B} \tilde{K} \tilde{C}$, it follows from Theorem 3.1 and Lemma 2.3 that there exists $\tau^{*}>0$ such that for every $\tau \in\left(0, \tau^{*}\right)$ the operator $\tilde{\Delta}_{\tau}$ is power stable. An application of Lemma 6.1 yields the claim.

Trivially, the compactness assumption on $\tilde{K} \tilde{C}$ is satisfied if the observation operators $C$ and $C_{c}$ are compact. In particular, compactness of $\tilde{K} \tilde{C}$ is guaranteed if $U$ and $Y$ are finite-dimensional.

Under suitable assumptions, Theorem 4.8 can be used to extend Theorem 6.2 to systems and controllers with unbounded control operators $B$ and $B_{c}$, respectively, provided the semigroups $T(t)$ and $T_{c}(t)$ are analytic. For the sake of brevity we omit the details.

\section{Appendix: Derivation of (4.54)}

Let $p \in(0, \pi)$ and consider the meromorphic function $g$ defined on $\mathbb{C}$ by

$$
g(\xi):=\frac{1+\xi-e^{\xi}}{\xi\left(e^{\xi}-1\right)} .
$$

Clearly, for any $\varepsilon \in(0,2 p)$, the function $g$ is bounded on $\mathbb{B}(0,2 p) \backslash \mathbb{B}(0, \varepsilon)$. Moreover, invoking the Taylor expansion of $e^{\xi}$ or using L'Hôpital's rule, we have

$$
\lim _{\xi \rightarrow 0} g(\xi)=-1 / 2 .
$$

Therefore, we may conclude that

$$
\sup \{|g(\xi)| \mid \xi \in \mathbb{B}(0,2 p), \quad \xi \neq 0\}<\infty .
$$


By a straightforward calculation it follows from (4.53) that

$$
\frac{|f(\tau, \lambda, s)|}{|\tau(s-\lambda)|}=\frac{|s|}{|s-\lambda|}|g(\tau(s-\lambda))| \text {. }
$$

Noting that

$$
\sup \left\{|s| /|s-\lambda| \mid(\lambda, s) \in \Gamma \times \mathbb{C}_{0}\right\}<\infty,
$$

we see from (A.1) that

$$
\sup \left\{\left|\frac{f(\tau, \lambda, s)}{\tau(s-\lambda)}\right| \mid(\tau, \lambda, s) \in(0, \infty) \times \Gamma \times \mathbb{C}_{0} \quad \text { s.t. } \tau|\lambda| \leq p, \tau|s| \leq p\right\}<\infty
$$

which is (4.54).

\section{REFERENCES}

[1] T. Chen and B. Francis, Input-output stability of sampled-data systems, IEEE Trans. Automat. Control 36 (1991), pp. 50-58. MR 91j:93094

[2] T. Chen and B. Francis, Optimal Sampled-Data Control Systems, Springer, London, 1995. MR 99a:93001

[3] R. F. Curtain and H. J. Zwart, An Introduction to Infinite-Dimensional Linear Systems Theory, Springer-Verlag, New York, 1995. MR 96i:93001

[4] N. Dunford and J. Schwartz, Linear Operators, Part II, John Wiley and Sons, New York, 1963. MR 32:6181

[5] K.-J. Engel and R. Nagel, One-Parameter Semigroups for Linear Evolution Equations, Graduate Texts in Mathematics, No. 194, Springer-Verlag, New York, 2000. MR 2000i:47075

[6] G. F. Franklin, J. D. Powell and M. Workman, Digital Control of Dynamic Systems, 3rd edition, Addison Wesley, Menlo Park, 1998.

[7] R. Kalman, B. Ho and K. Narendra, Controllability of linear dynamical systems, Contributions to Differential Equations 1 (1963), pp. 188-213. MR 27:5012

[8] T. Kato, Perturbation Theory for Linear Operators, 2nd edition, Springer-Verlag, Berlin, 1980. MR 96a:47025

[9] H. Logemann, Stability and stabilizability of linear infinite-dimensional discrete-time systems, IMA J. of Mathematical Control \& Information 9 (1992), pp. 255-263. MR 94c:93094

[10] I. Lasiecka and R. Triggiani, Stabilization and structural assignment of Dirichlet boundary feedback parabolic equations, SIAM J. Control 21 (1983), pp. 766-803. MR 85i:93028

[11] I. Lasiecka and R. Triggiani, The regulator problem for parabolic equations with Dirichlet boundary control, Part I: Riccati's feedback synthesis and regularity of optimal solution, Appl. Math. Optim. 16 (1987), pp. 147-168. MR 88g:93063a

[12] I. Lasiecka and R. Triggiani, The regulator problem for parabolic equations with Dirichlet boundary control, Part II: Galerkin approximation, Appl. Math. Optim. 16 (1987), pp. 187216. MR 88g:93063b

[13] J.-L. Lions and E. Magenes, Non-homogeneous Boundary Value Problems and Applications, Vols. 1 and 2, Springer, Berlin, 1972. MR 50:2670 MR 50:2671

[14] A. Pazy, Semigroups of Linear Operators and Applications to Partial Differential Equations, Applied Mathematical Sciences 44, Springer-Verlag, New York, 1983. MR 85g:47061

[15] R. Rebarber and S. Townley, Stabilization of distributed parameter systems by piecewise polynomial control, IEEE Trans. Automat. Control 42 (1997), pp. 1254-1257. MR 98j:93073

[16] R. Rebarber and S. Townley, Generalized sampled data feedback control of distributed parameter systems, Systems \& Control Letters 34 (1998), pp. 229-240. MR 99f:93081

[17] R. Rebarber and S. Townley, Non-robustness of closed-loop stability for infinite-dimensional systems under sample and hold, IEEE Trans. Automat. Control 47 (2002), pp. 1381-1385.

[18] I. G. Rosen and C. Wang, On stabilizability and sampling for infinite-dimensional systems, IEEE Trans. Automat. Control 37 (1992), pp. 1653-1656. MR 93g:93077

[19] E. D. Sontag, Mathematical Control Theory, 2nd edition, Springer-Verlag, New York, 1998. MR 99k:93001

[20] T. J. Tarn, J. R. Zavgren and X. M. Zeng, Stabilization of infinite-dimensional systems with periodic gains and sampled output, Automatica, 24 (1988), pp. 95-99. MR 89d:93087 
[21] G. Weiss, Admissibility of unbounded control operators, SIAM J. Control \& Optim. 27 (1989), pp. 527-545. MR 90c:93060

[22] Y. Yamamoto, A function space approach to sampled data control systems and tracking problems, IEEE Trans. Automat. Control 39 (1994), pp. 703-713. MR 95b:93116

[23] J. Zabczyk. On decomposition of generators, SIAM J. Control \& Optim. 16 (1978), pp. 523-534 (erratum in SIAM J. Control \& Optim. 18 (1980), p. 325). MR 58:23757] MR 81c: 47044

Department of Mathematical Sciences, University of Bath, Bath BA2 7AY, United KINGDOM

E-mail address: hl@maths.bath.ac.uk

Department of Mathematics and Statistics, University of Nebraska-Lincoln, LinCOLN, NeBRASKa 68588-0323

E-mail address: rrebarbe@math.unl.edu

School of Mathematical Sciences, University of Exeter, Exeter, EX4 4QE, United KINGDOM

E-mail address: townley@maths.ex.ac.uk 